\title{
Development of QSAR models using artificial neural network analysis for risk assessment of repeated-dose, reproductive, and developmental toxicities of cosmetic ingredients
}

\author{
Tomoka Hisaki, Maki Aiba née Kaneko, Masahiko Yamaguchi, Hitoshi Sasa \\ and Hirokazu Kouzuki
}

Shiseido Research Center, Shiseido Co. Ltd., 2-2-1 Hayabuchi, Tsuzuki-ku, Yokohama-shi, Kanagawa 224-8558, Japan

(Received November 13, 2014; Accepted December 30, 2014)

\begin{abstract}
Use of laboratory animals for systemic toxicity testing is subject to strong ethical and regulatory constraints, but few alternatives are yet available. One possible approach to predict systemic toxicity of chemicals in the absence of experimental data is quantitative structure-activity relationship (QSAR) analysis. Here, we present QSAR models for prediction of maximum "no observed effect level" (NOEL) for repeated-dose, developmental and reproductive toxicities. NOEL values of 421 chemicals for repeated-dose toxicity, 315 for reproductive toxicity, and 156 for developmental toxicity were collected from Japan Existing Chemical Data Base (JECDB). Descriptors to predict toxicity were selected based on molecular orbital (MO) calculations, and QSAR models employing multiple independent descriptors as the input layer of an artificial neural network (ANN) were constructed to predict NOEL values. Robustness of the models was indicated by the root-mean-square (RMS) errors after 10-fold cross-validation ( 0.529 for repeated-dose, 0.508 for reproductive, and 0.558 for developmental toxicity). Evaluation of the models in terms of the percentages of predicted NOELs falling within factors of 2, 5 and 10 of the in-vivo-determined NOELs suggested that the model is applicable to both general chemicals and the subset of chemicals listed in International Nomenclature of Cosmetic Ingredients (INCI). Our results indicate that ANN models using in silico parameters have useful predictive performance, and should contribute to integrated risk assessment of systemic toxicity using a weight-of-evidence approach. Availability of predicted NOELs will allow calculation of the margin of safety, as recommended by the Scientific Committee on Consumer Safety (SCCS).
\end{abstract}

Key words: Artificial neural network, Repeated-dose toxicity, Reproductive/developmental toxicity, QSAR model, NOEL, Cosmetic ingredients

\section{INTRODUCTION}

Many well-validated and proven in vivo assays are available for systemic toxicity testing. Repeated-dose toxicity refers to general toxicological effects in mammals occurring as a result of prolonged and repeated exposure to a substance, and covers a wide range of possible adverse effects, including changes in morphology, physiology, growth, development or life span. The most commonly performed animal tests for repeated-dose toxicity are 28-day and 90-day oral toxicity tests in rodents. Other major endpoints for systemic toxicity are reproductive toxicity and developmental toxicity, which refer to the adverse effects of substances on sexual function and fer- tility in adult males and females, and developmental toxicity in offspring (including effects on, or mediated via, lactation), respectively. In this study, we define reproductive toxicity in terms of a range of endpoints relating to impairment of male and female fertility, and we define developmental toxicity in terms of adverse effects on offspring. Studies on reproductive toxicity and developmental toxicity usually aim to identify adverse effects on various organs and tissues, and to establish the lowest dose that induces an effect (lowest observed (adverse) effect level; LO(A)EL) or the highest dose with no biologically or statistically significant effect (no observed (adverse) effect level; $\mathrm{NO}(\mathrm{A}) \mathrm{EL})$.

Laboratory animals are generally used for toxici-

Correspondence: Tomoka Hisaki (E-mail: tomoka.hisaki@to.shiseido.co.jp) 


\section{T. Hisaki et al.}

ty testing, especially for reproductive and developmental toxicity (Jagt et al., 2004; Hartung and Rovida, 2009; Spielmann and Vogel, 2006). But, according to the 7th amendment of the EU cosmetic directive, cosmetic products containing substances that have been tested in animals may no longer be sold in the EU. Nevertheless, the importance of systemic toxicity assessment for cosmetic ingredients has been highlighted in "Notes of Guidance for Testing of Cosmetic Ingredients and Their Safety Evaluation" by the Scientific Committee on Consumer Safety (SCCS) as well as in "Safety Evaluation Guidelines Edition 2014" by the The Personal Care Products Council (PCPC), which both strongly recommends calculation of the margin of safety; for this purpose, NOEL data for repeated-dose, reproductive, and developmental toxicities are required. Therefore, non-animal test methods for assessing systemic toxicity are necessary; i.e., we require methods to predict NOEL of chemicals.

At present, in vitro methods for predicting developmental toxicity have advanced furthest towards regulatory acceptance. Indeed, the embryonic stem cell test (EST; Spielmann et al., 1997) is the only in vitro method that has been validated by The European Centre for the Validation of Alternative Methods (ECVAM; Brown et al., 1995; Seiler and Spielmann et al., 2011). However, because of the multiplicity and complexity of the processes underlying systemic toxicity, no method has yet been brought into general use.

A different approach would be to use in silico methods. The two major methodologies for in silico assessment of toxicity are (quantitative) structure-activity relationship ((Q)SAR) analysis and read-across. (Q)SAR models are built to find mathematical relationships between physicochemical or structural properties and biological activity, based on the idea that chemical structural characteristics are related to biological activity. The most widely used QSAR software for quantitative prediction of repeated-dose toxicity is Toxicity Prediction by Komputer Assisted Technology (TOPKAT; Accelrys, San Diego, CA, USA). TOPKAT is designed to predict rat chronic oral LOAEL based on a dataset of 393 chemicals collected from the U.S. Environmental Protection Agency (EPA), National Cancer Institute/National Toxicology Program (NCI/NTP), National Toxicology Program Technical Reports, Food and Drug Administration New Drug Applications, and literature reports (Mumtaz et al., 1995; Venkatapathy et al., 2004). Several studies (Mumtaz et al., 1995; Venkatapathy et al., 2004; Tilaoui et al., 2007; Rupp et al., 2010) have found that reasonable predictions can be obtained for a range of chemicals. Another recently developed model is MolCode Toolboxes (MolCode,
Ltd., Tartu, Estonia). However, both models are designed to predict LOAEL, and cannot directly predict NOEL, which is needed for calculation of the margin of safety.

For developmental or reproductive toxicity, there are some software tools based on QSAR (reviewed in Cronin and Worth, 2008; Piparo and Worth, 2010), but these were constructed to predict hazards related to developmental or reproductive toxicity (i.e., they are limited to qualitative analysis) and no quantitative model has been reported.

The aim of the present study was to construct QSAR models enabling the prediction of NOELs for repeateddose, developmental and reproductive toxicities by combining a molecular orbital (MO) calculation method and an artificial neural network (ANN) system. Although a single model is unlikely to be sufficient for assessment of systemic toxicity, the present study indicates that prediction of NOELs using an in silico approach may be feasible. This would enable calculation of the margin of safety, which would be very important for safety assessment of cosmetic raw materials according to the weight of evidence approach.

\section{MATERIAL AND METHODS}

\section{Toxicological data}

NOELs of repeated-dose toxicity and developmental/ reproductive toxicity in rats were collected from the Japan Existing Chemical Data Base (JECDB; http:// dra4.nihs.go.jp/mhlw_data/jsp/SearchPage.jsp). For repeated-dose toxicity, NOELs obtained from repeateddose 28-day (or 90-day) oral toxicity study or combined repeated-dose toxicity study and reproductive and developmental toxicity screening of 421 chemicals were collected. For reproductive toxicity, NOELs of 315 chemicals for parents were collected, while for developmental toxicity, NOELs of 156 chemicals for offspring were collected. NOELs obtained from combined repeated-dose toxicity study with reproductive and developmental toxicity screening or from reproductive and developmental toxicity screening alone were included (Table 1). However, NOEL of general toxicity obtained in reproductive and developmental toxicity screening was not used. It should be noted that $1000 \mathrm{mg} / \mathrm{kg}$ was the maximum dose in these studies. Chemicals which showed toxicity at the lowest dose of the test, i.e. NOEL was not obtained, were not included.

\section{Extraction of descriptors}

The structures of chemicals were built with Chem 3D Ultra 10.0 (CambridgeSoft Co., Cambridge, MA, USA) and modeled in their neutral forms. Geometry optimiza- 
QSAR prediction of systemic toxicity of cosmetic ingredients

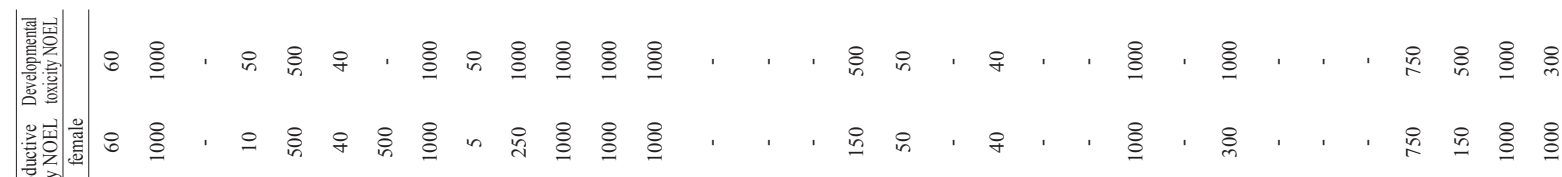

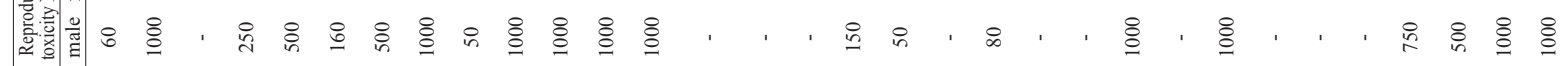

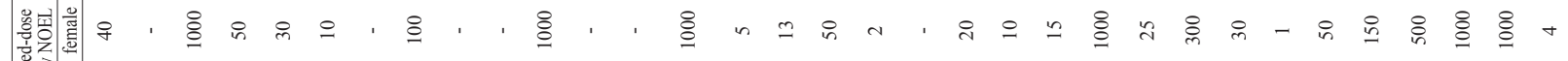
密:

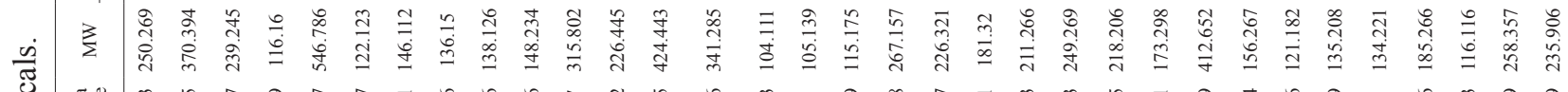

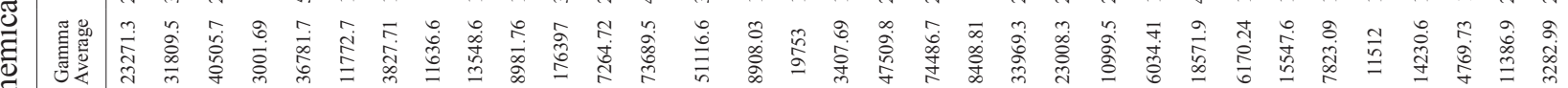

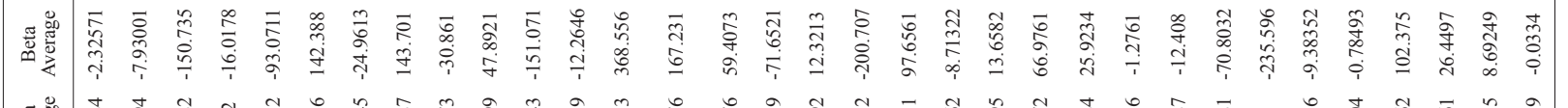

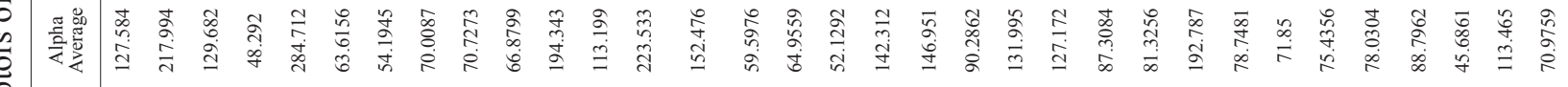

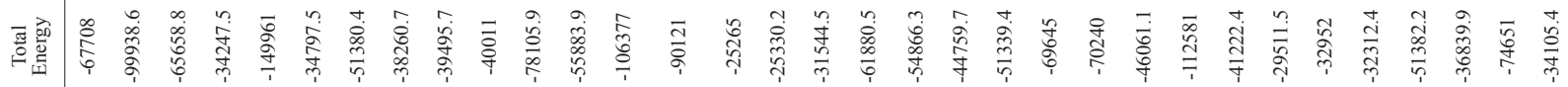

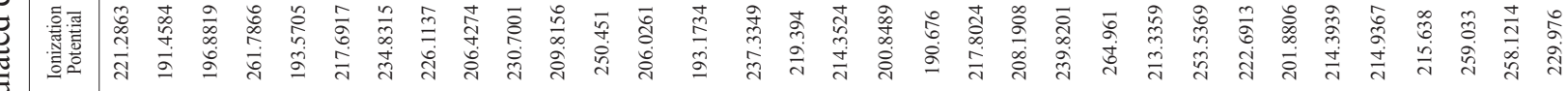

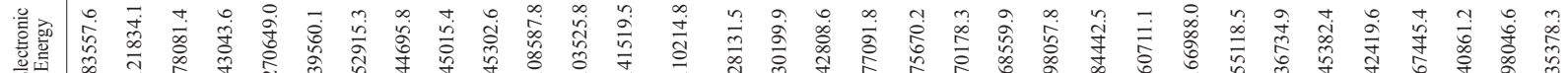

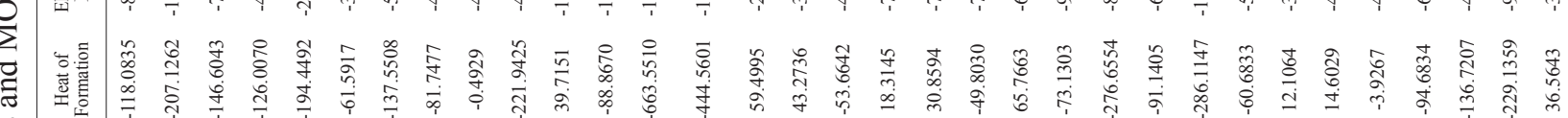

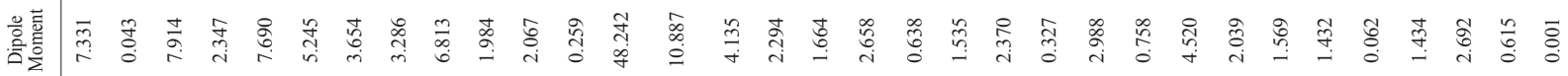

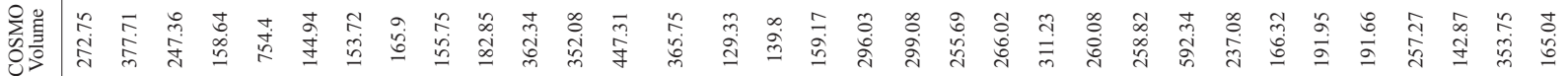

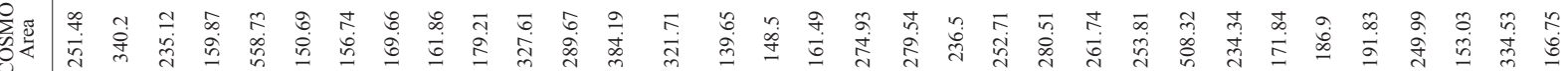

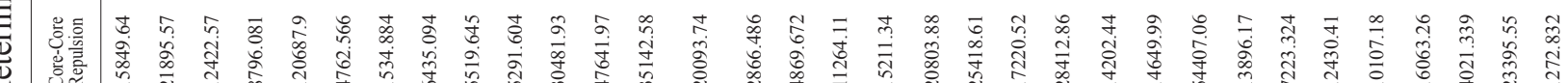

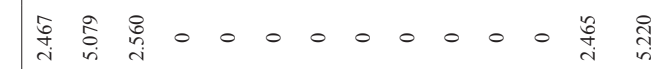




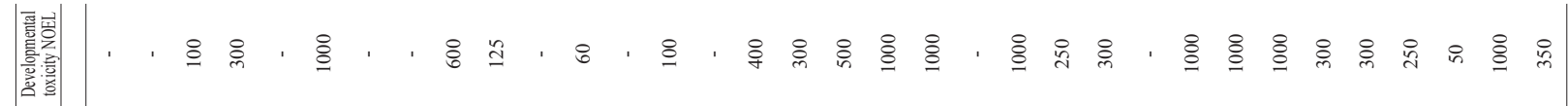

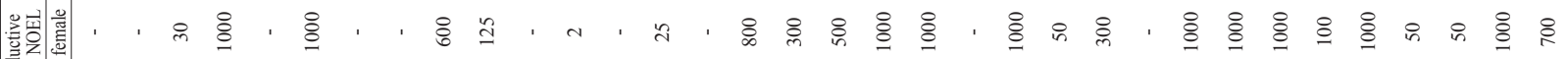

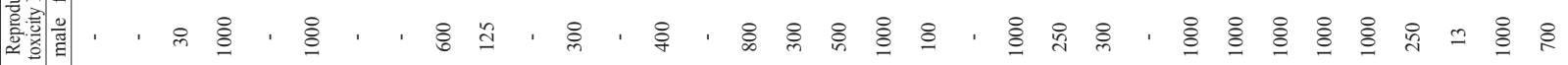

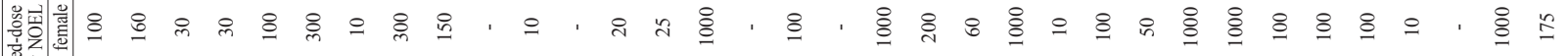

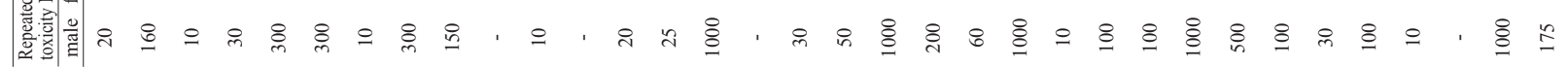

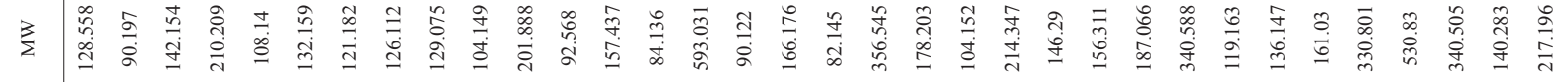

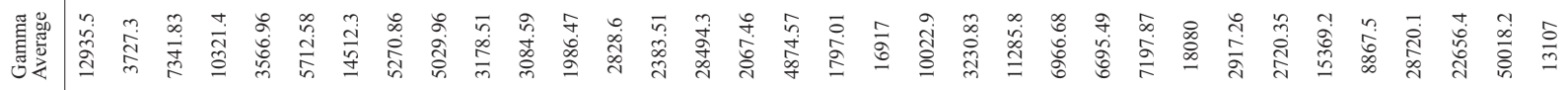

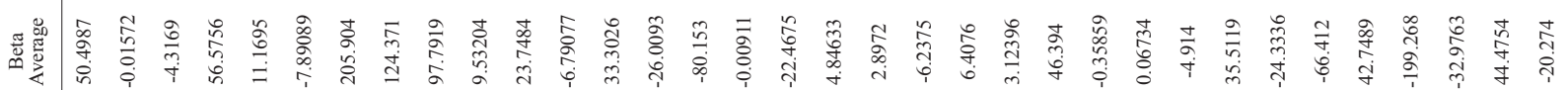

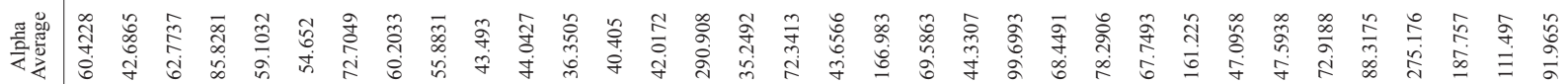

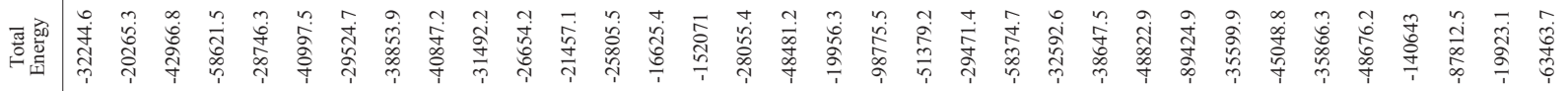

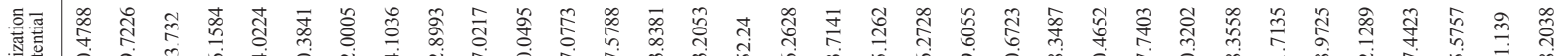

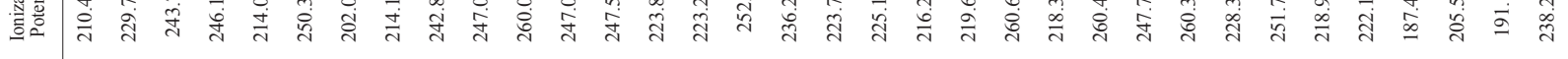

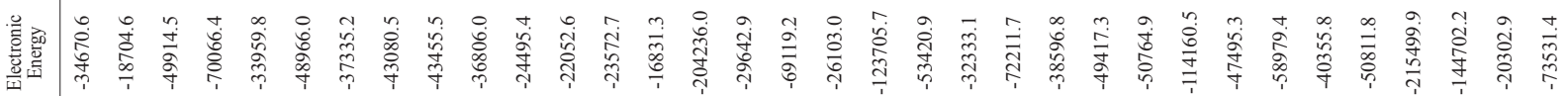

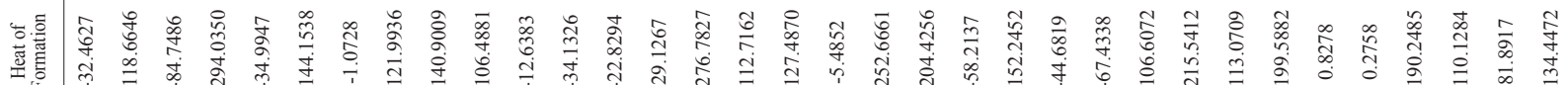

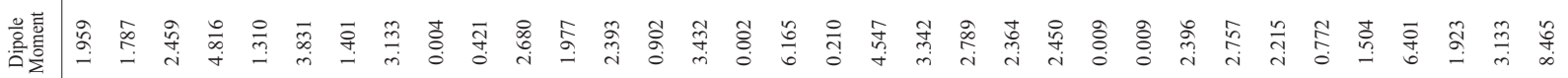

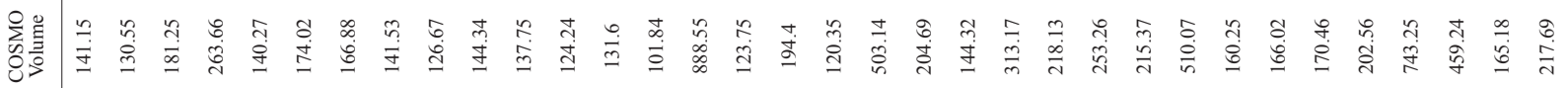

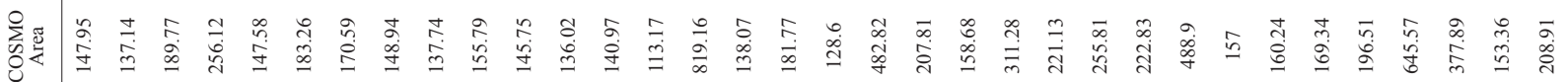

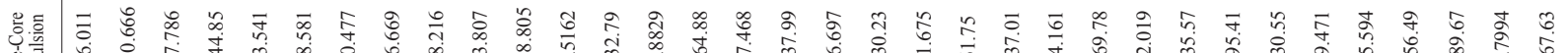

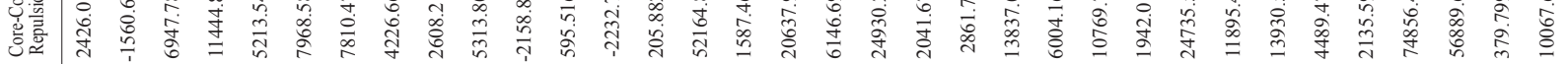

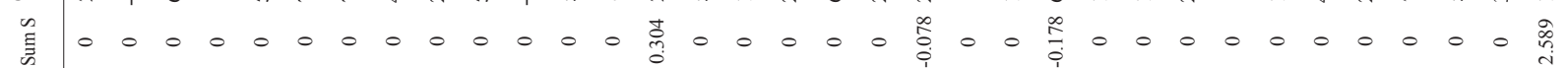

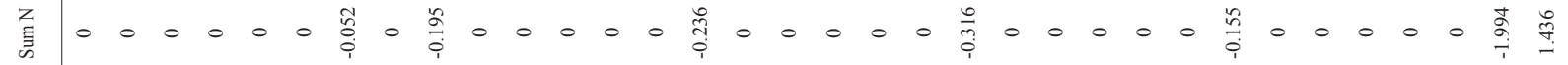

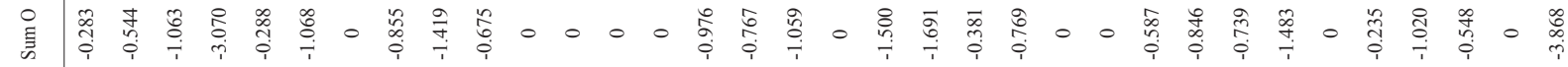

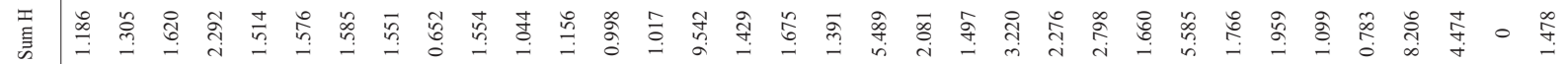

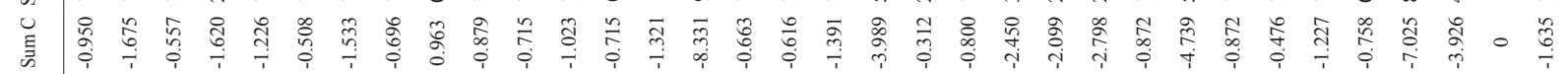

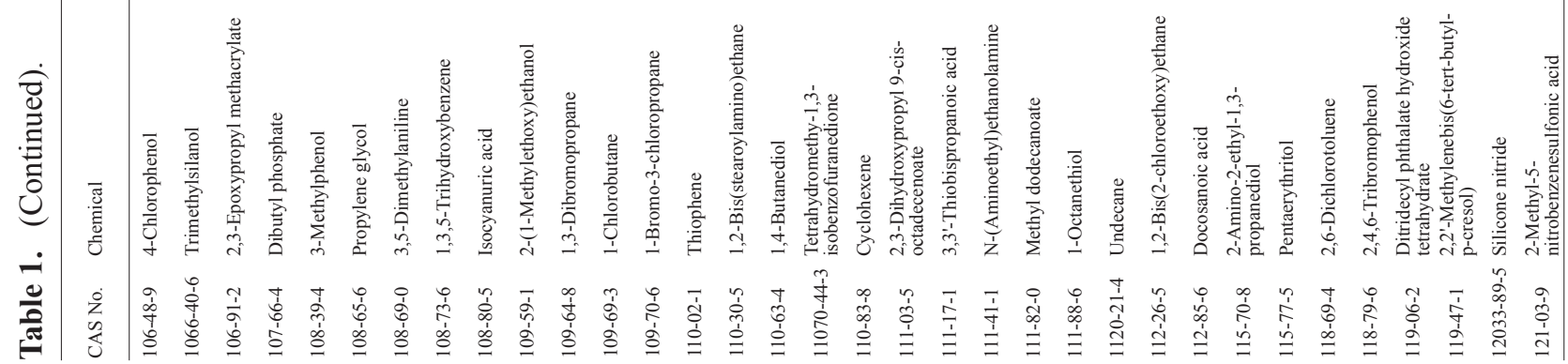

Vol. 40 No. 2 
QSAR prediction of systemic toxicity of cosmetic ingredients

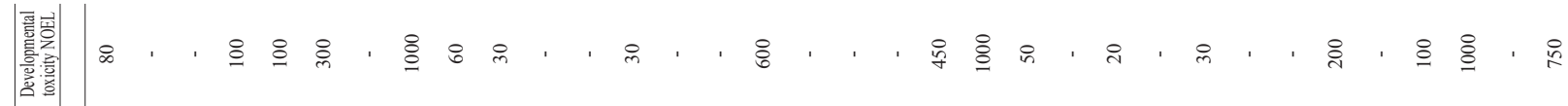

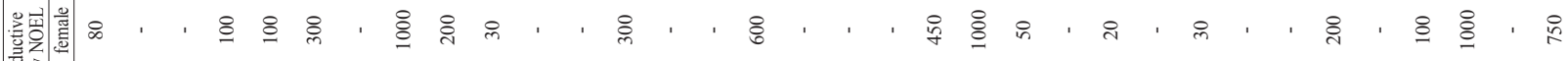

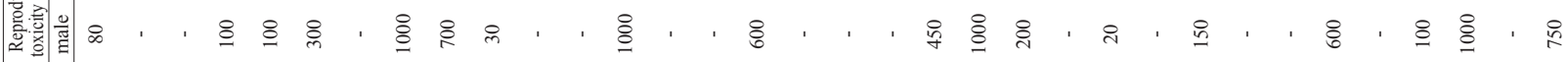

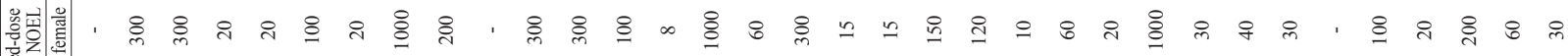

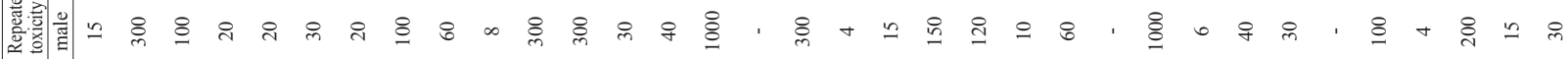

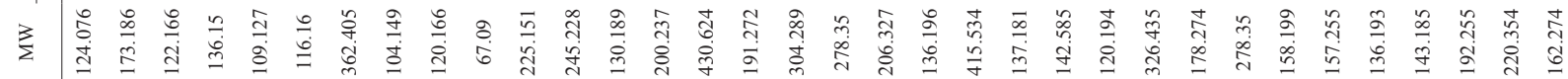

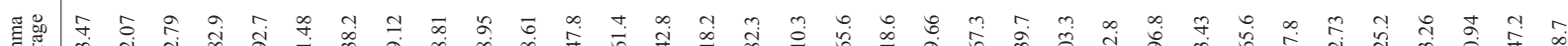

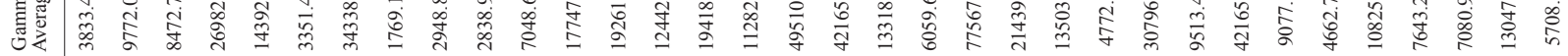

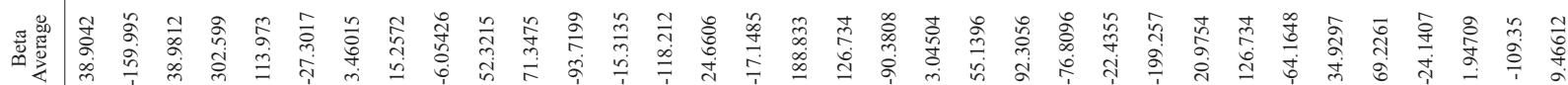

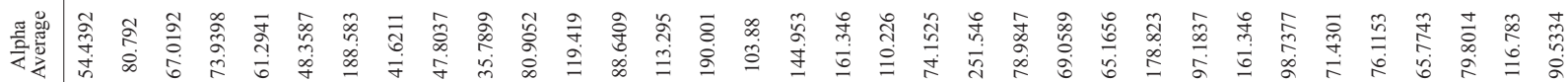

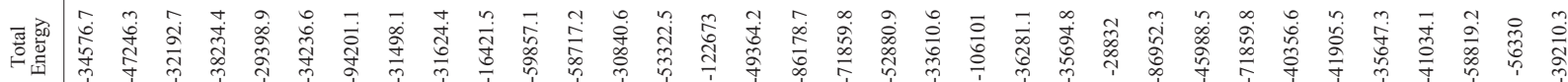

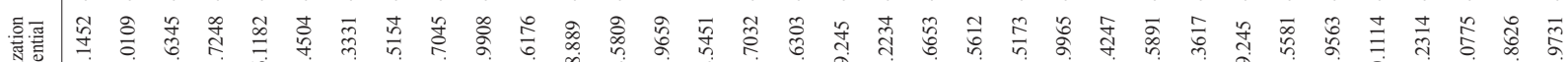

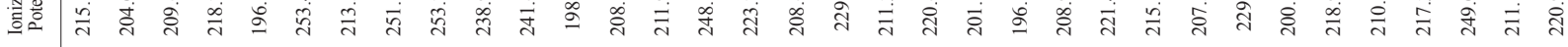

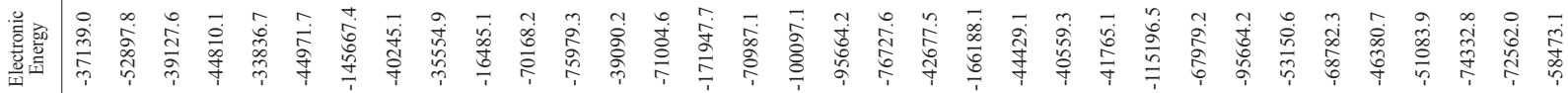

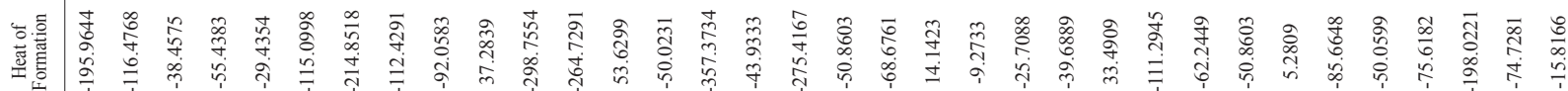

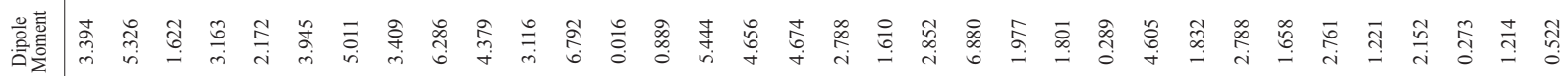

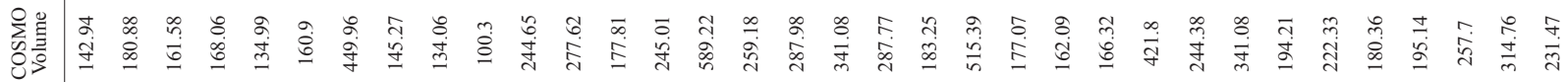

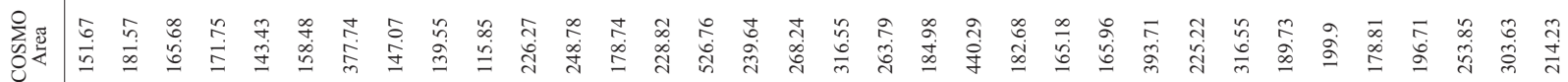

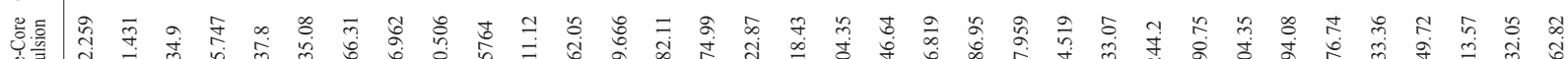

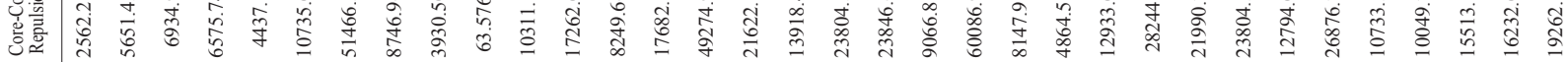

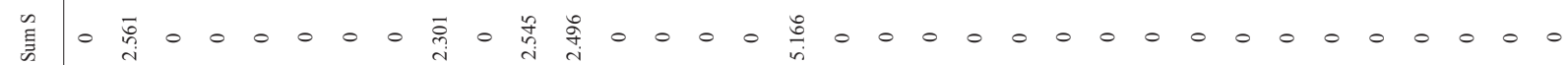

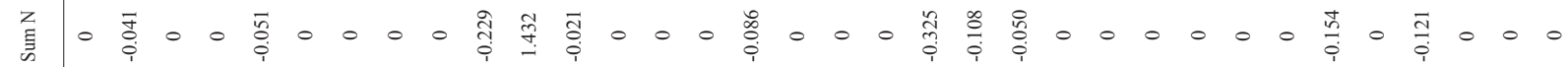

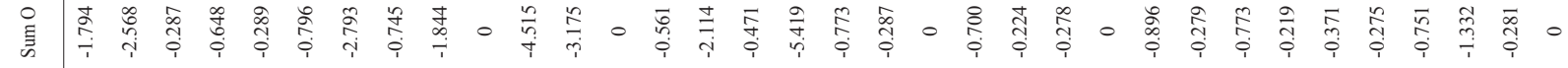
王 竞

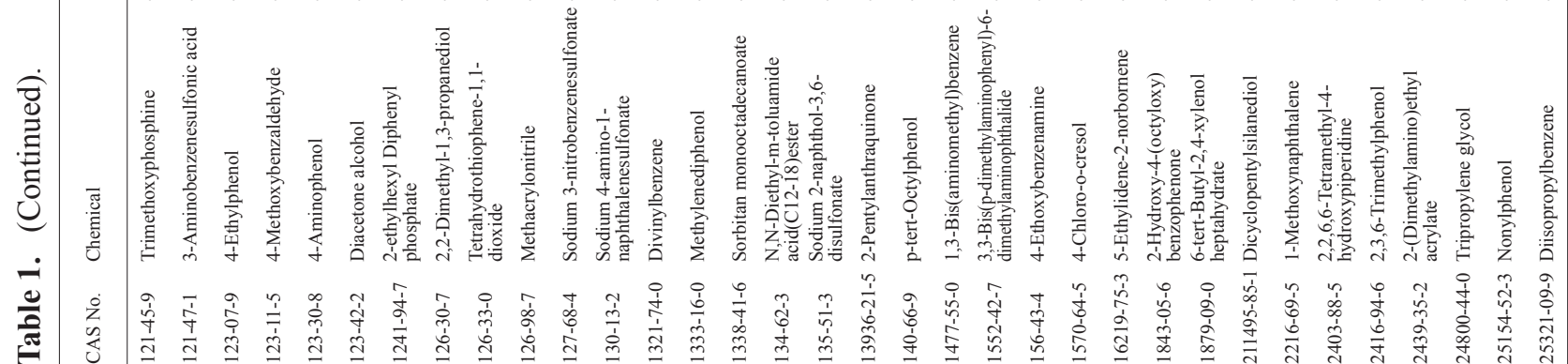




\section{T. Hisaki et al.}

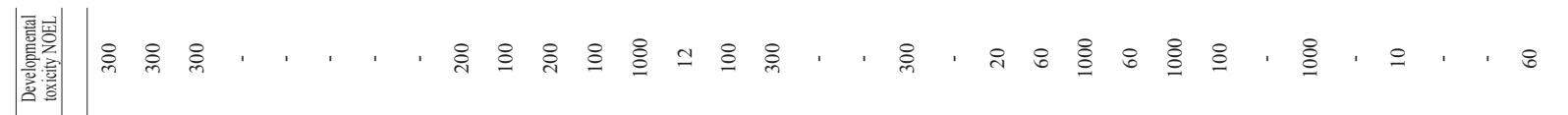

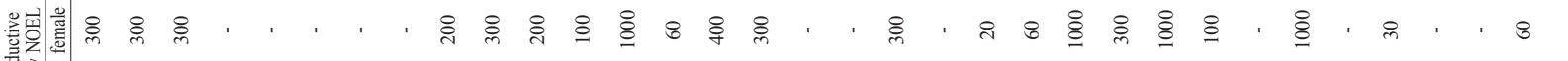

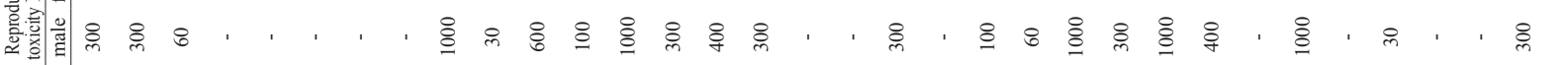

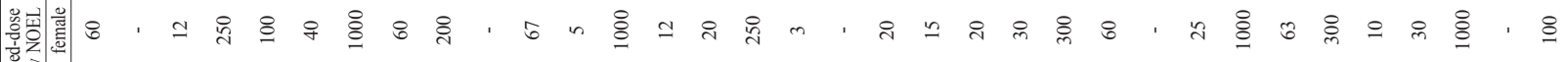

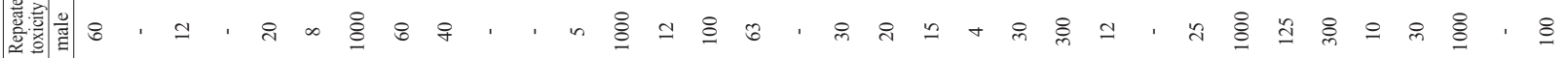

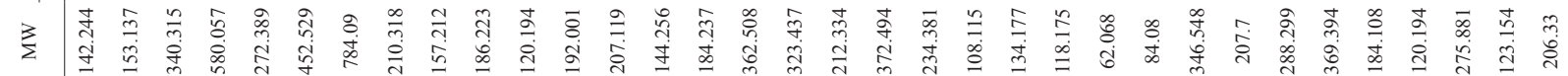

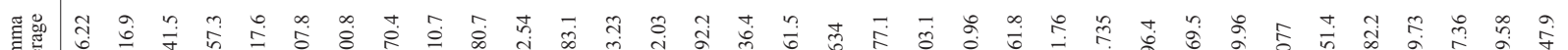

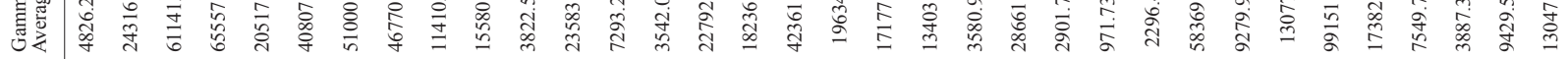

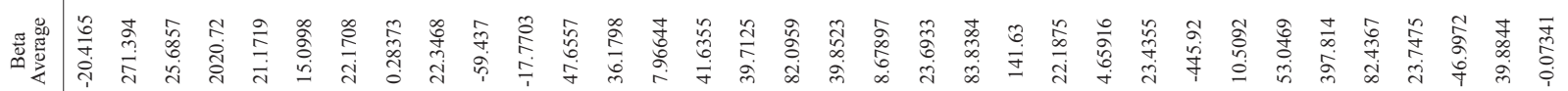

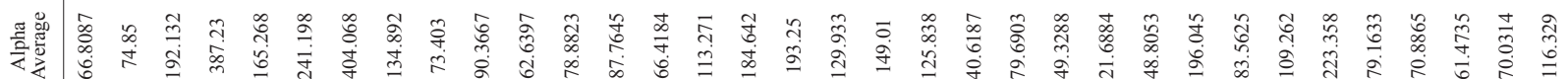

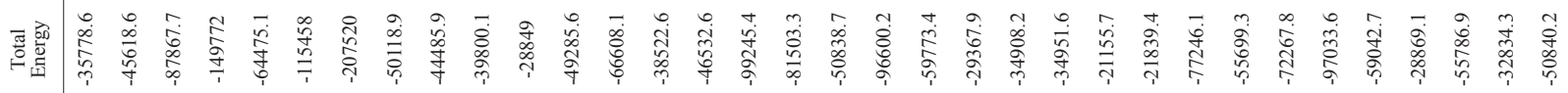

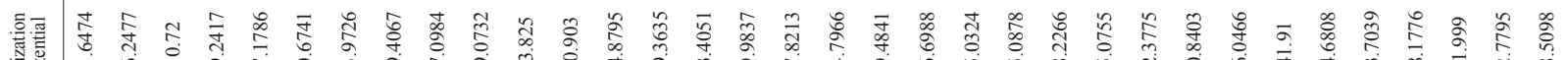

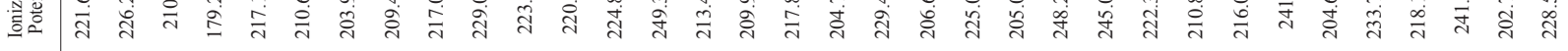

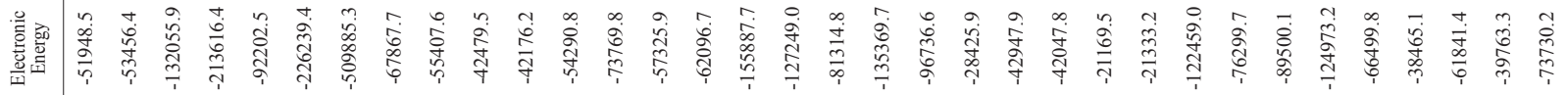

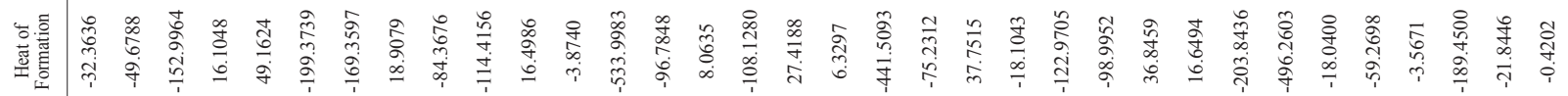

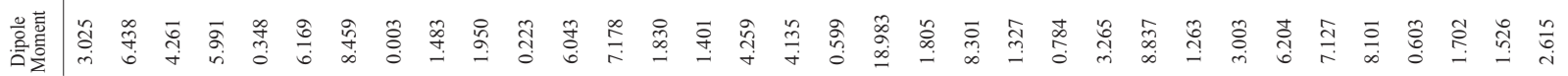

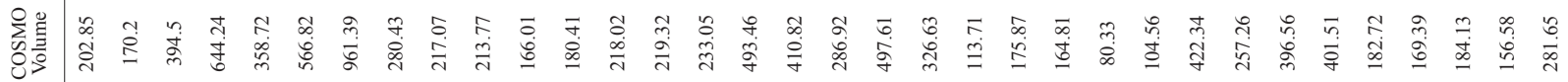

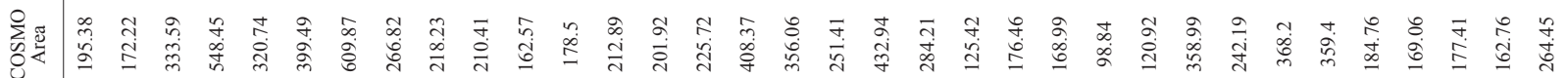

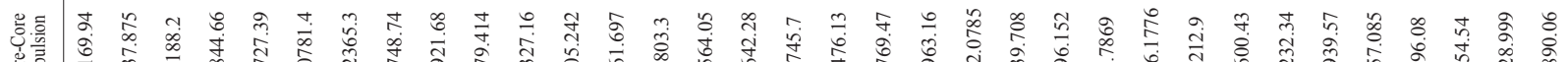

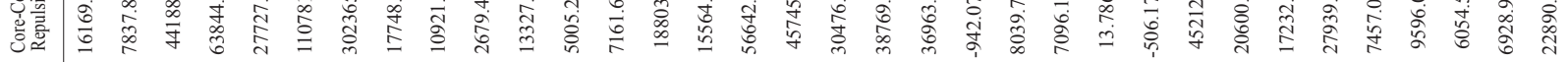

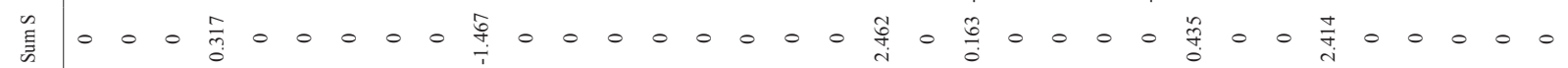

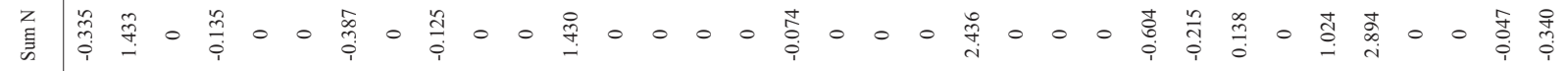

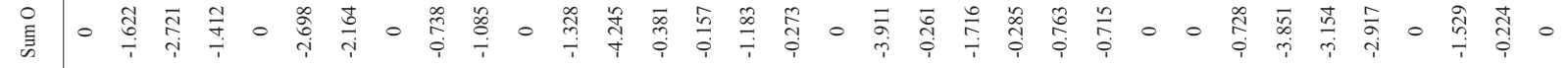

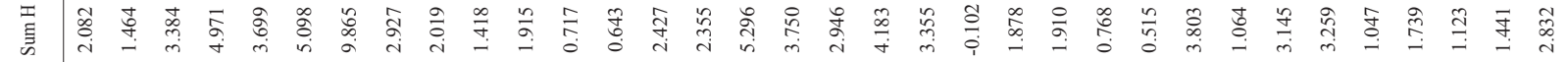

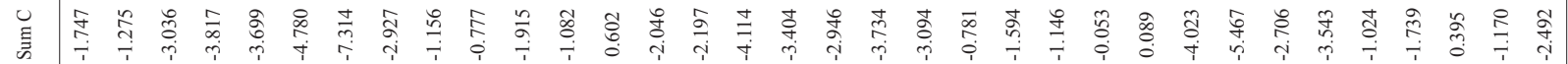

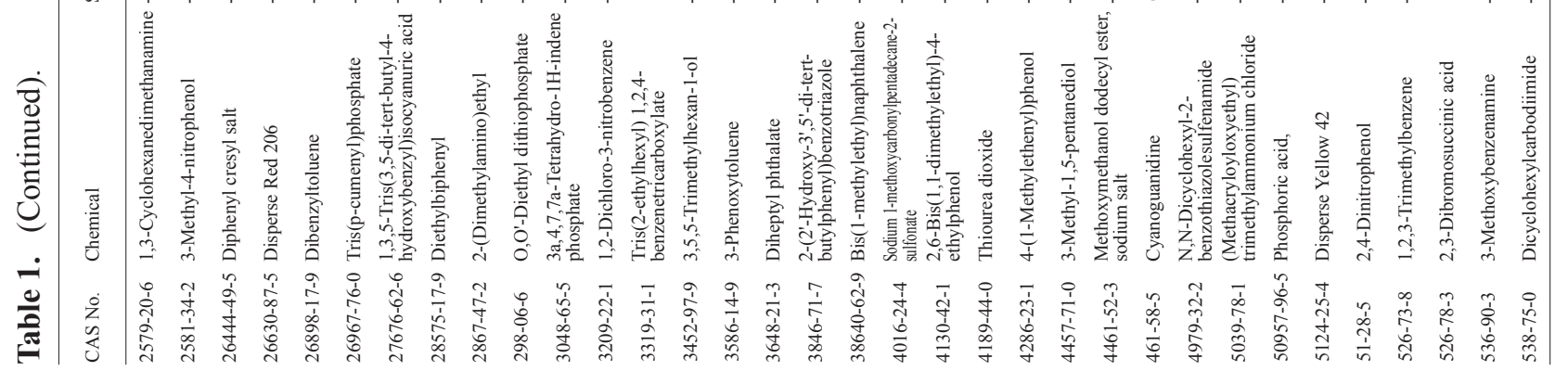

Vol. 40 No. 2 
QSAR prediction of systemic toxicity of cosmetic ingredients

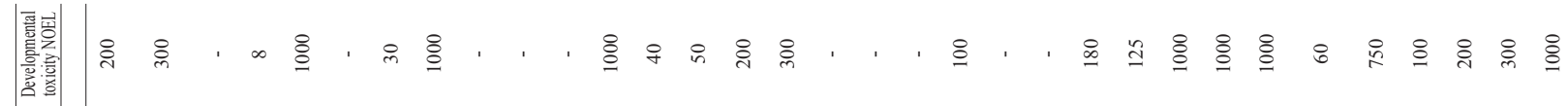

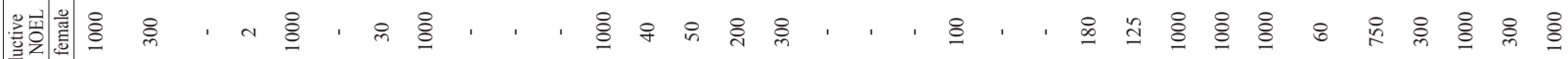

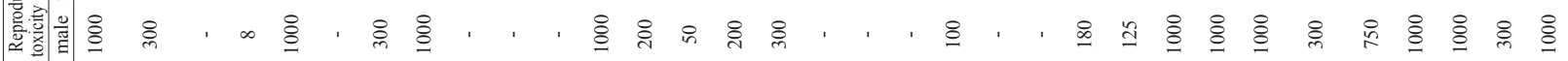

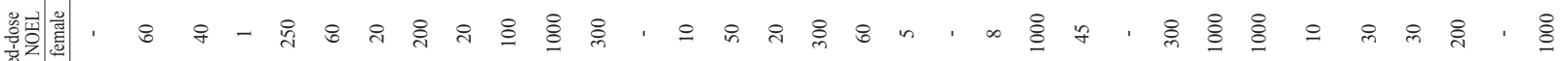

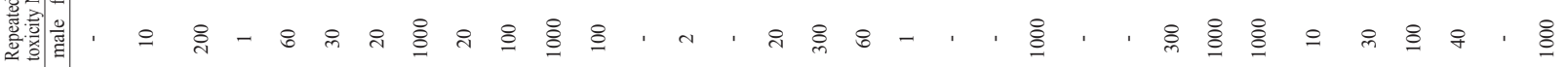

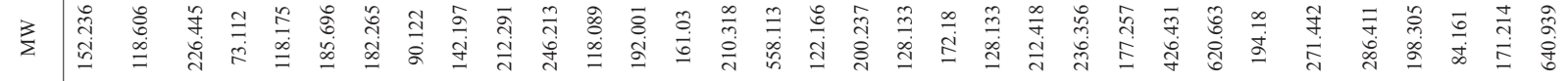

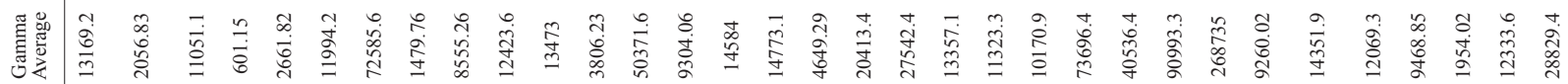

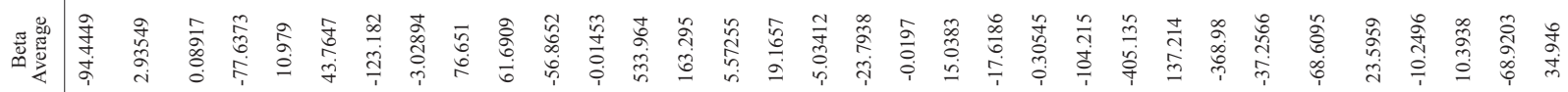

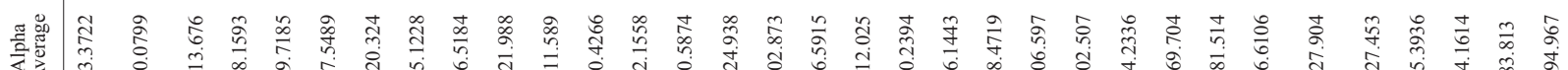

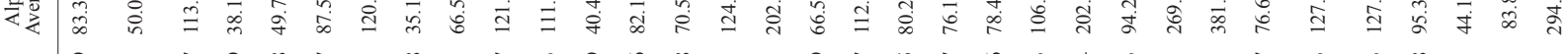

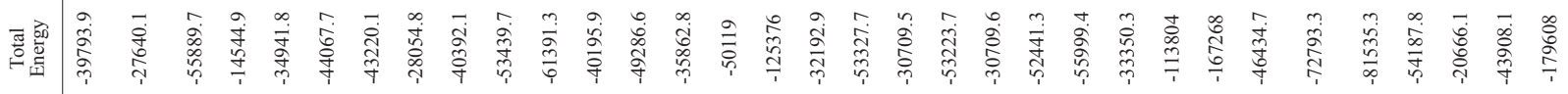

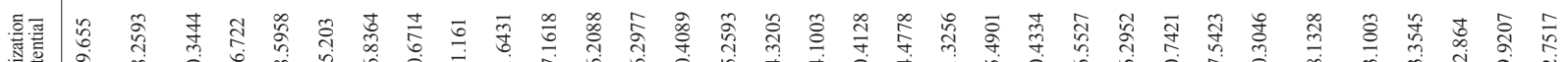

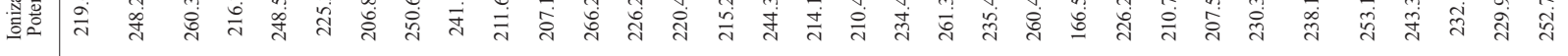

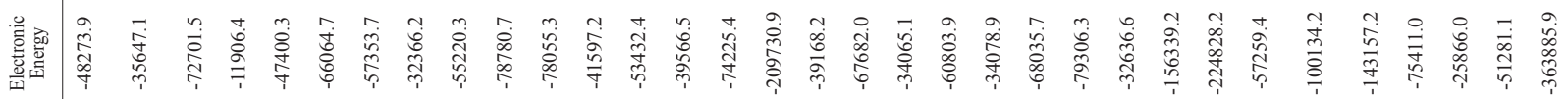

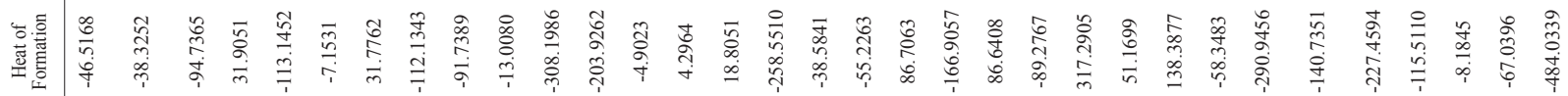

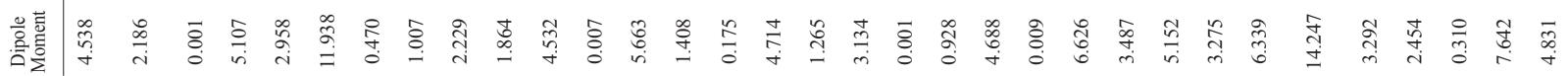

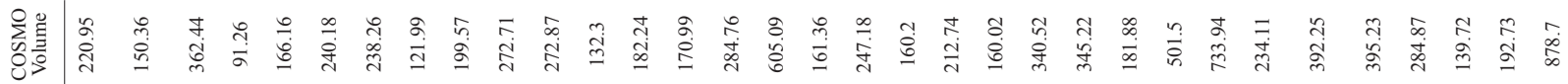

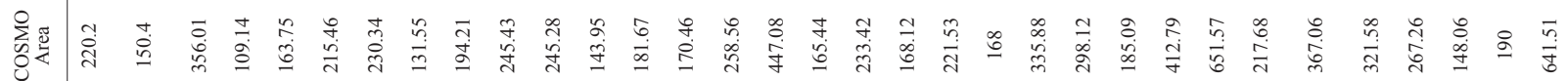

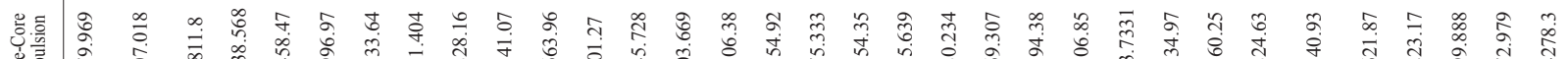

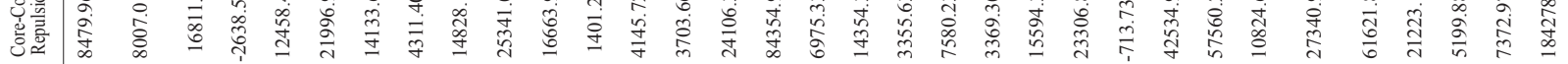

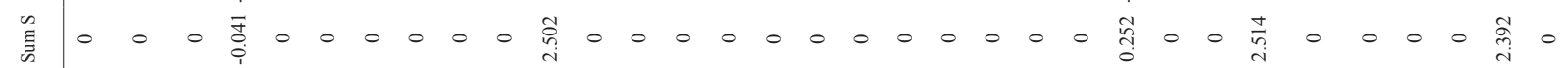

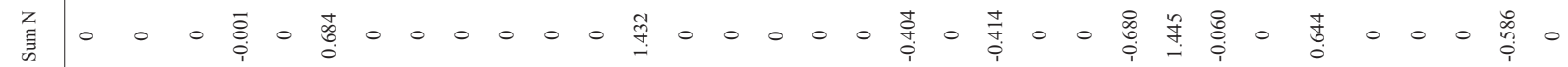

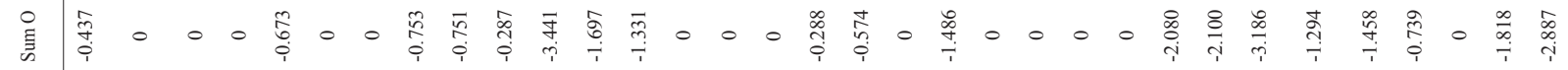

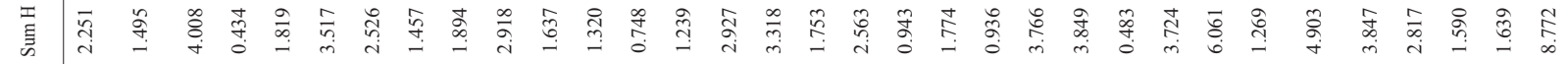

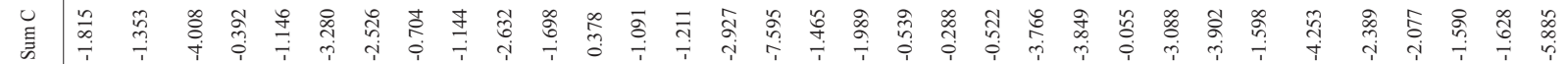

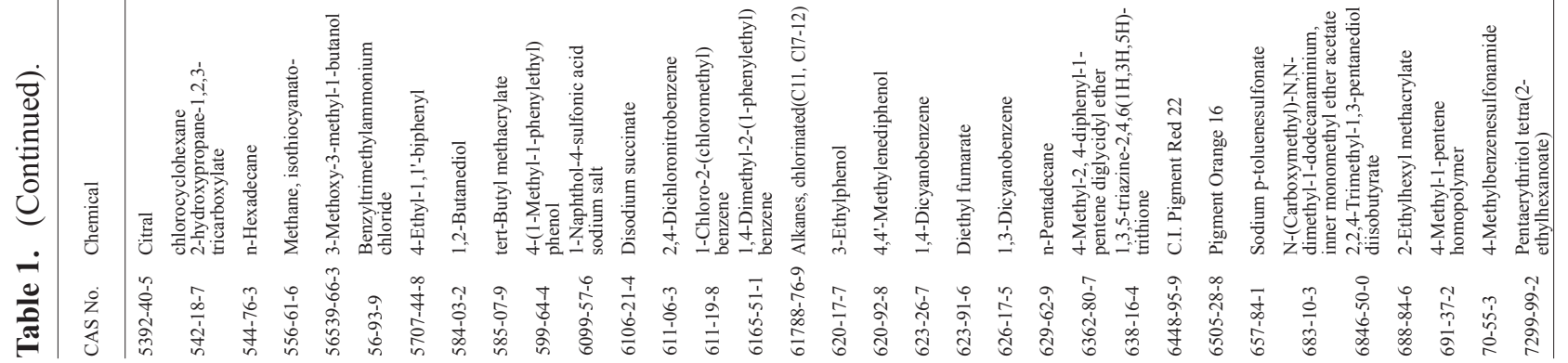


T. Hisaki et al.

|

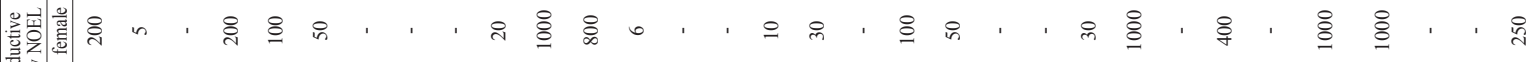

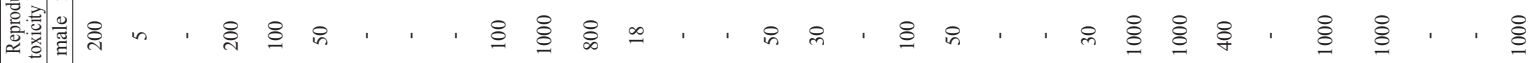

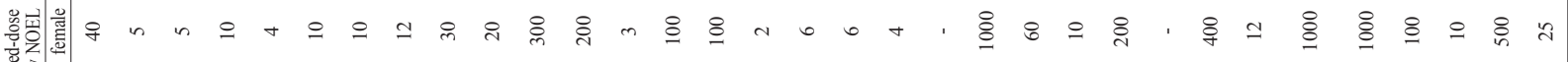

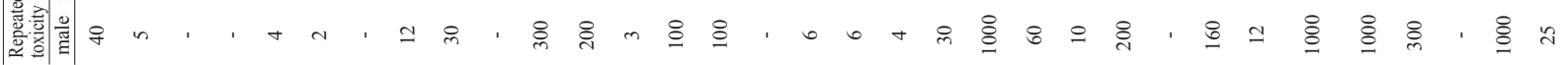

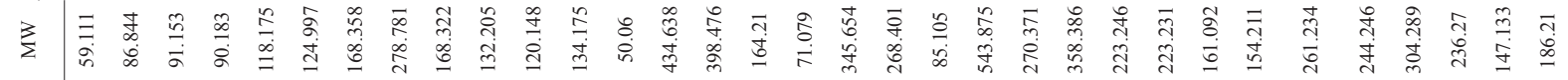

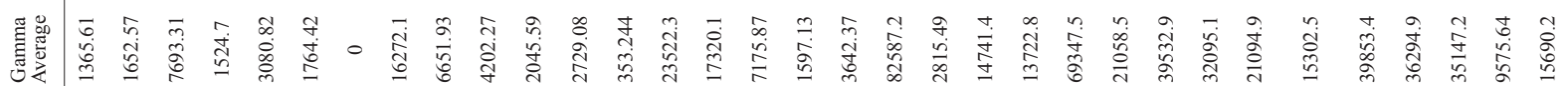

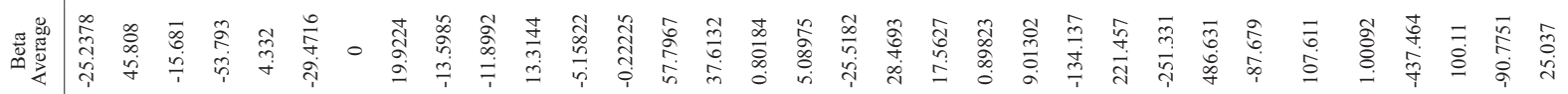

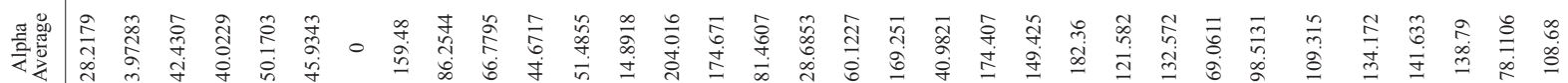

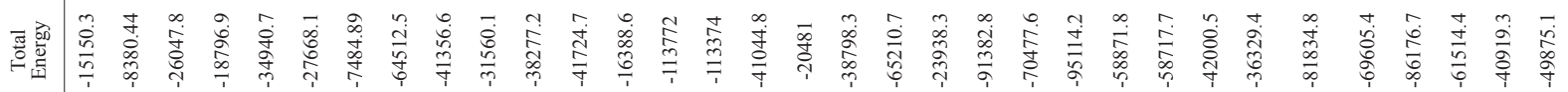

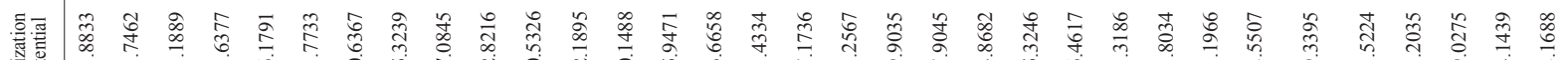

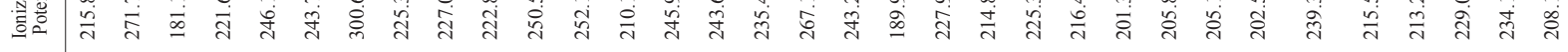

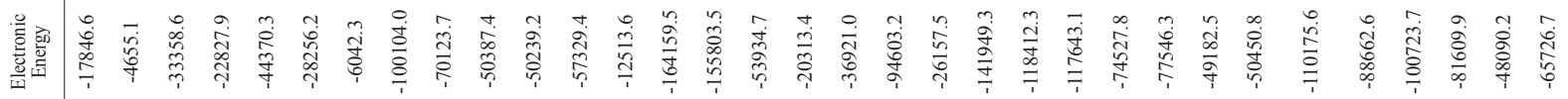

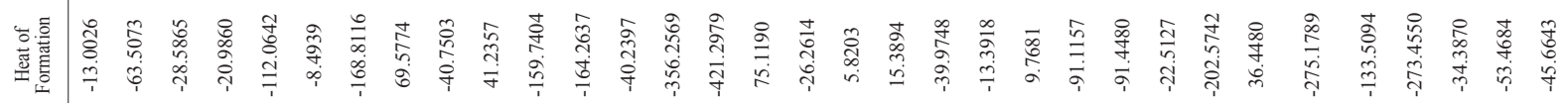

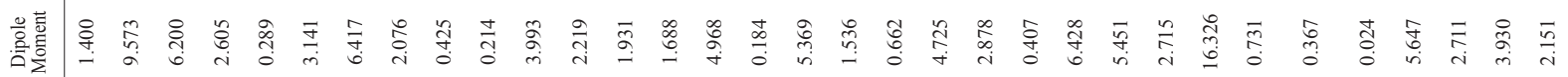

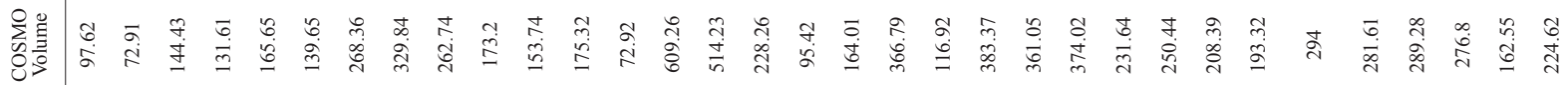

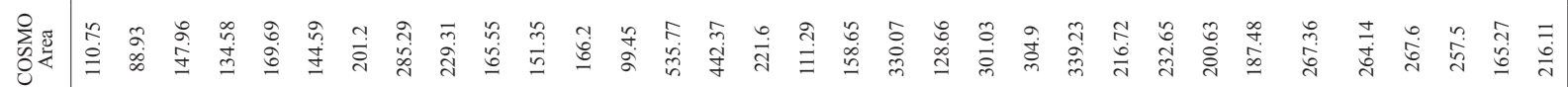

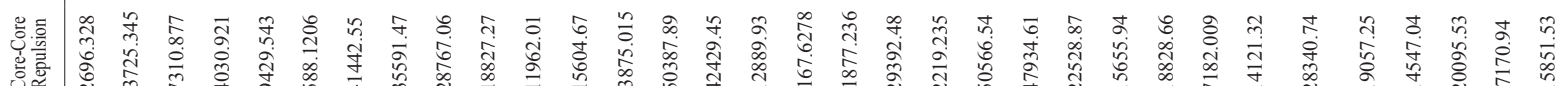

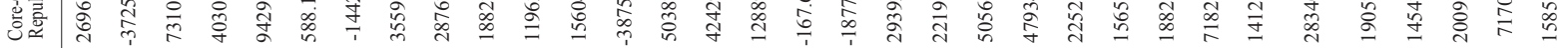

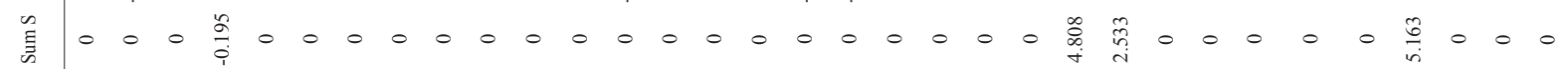

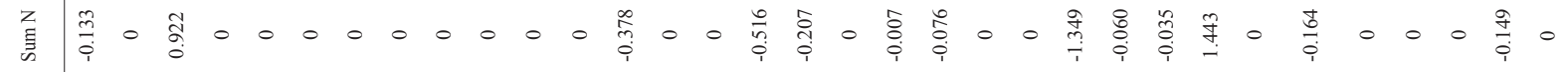

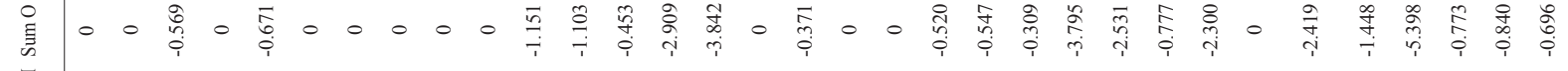

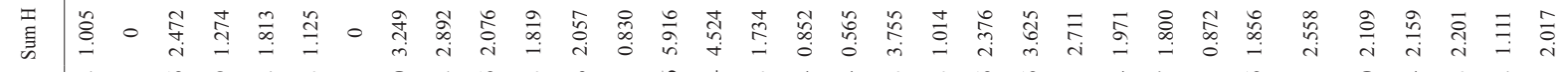

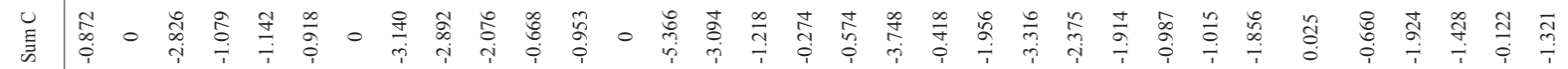

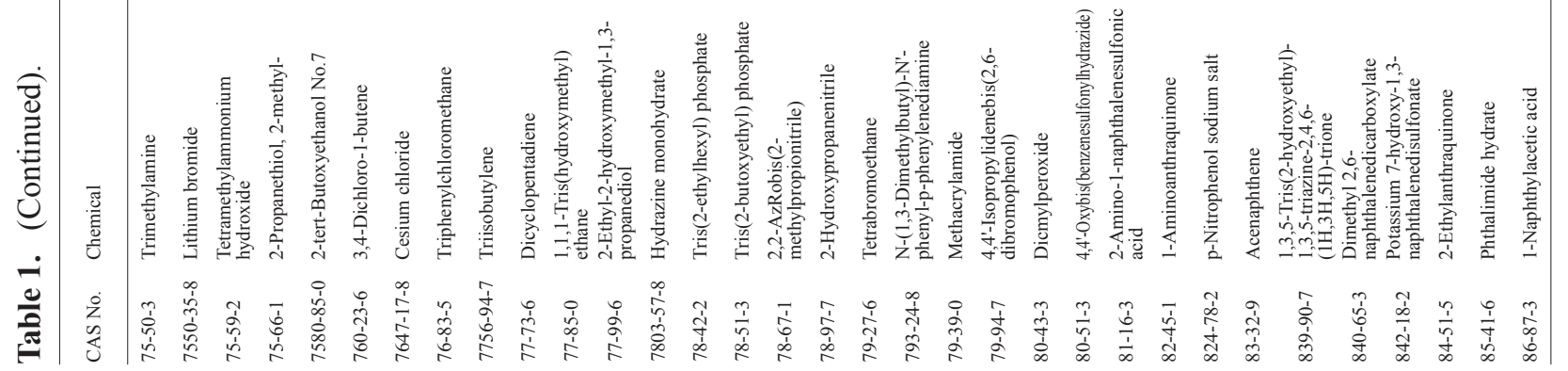

Vol. 40 No. 2 
QSAR prediction of systemic toxicity of cosmetic ingredients

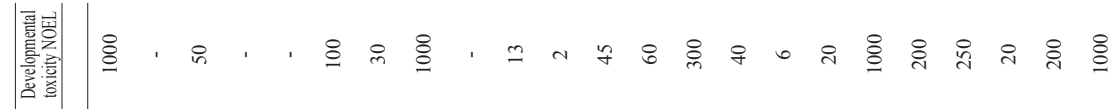

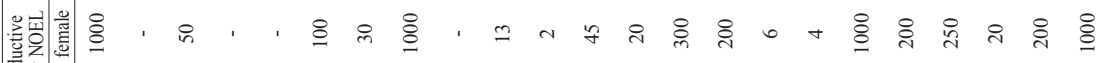

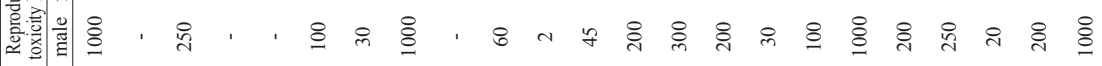

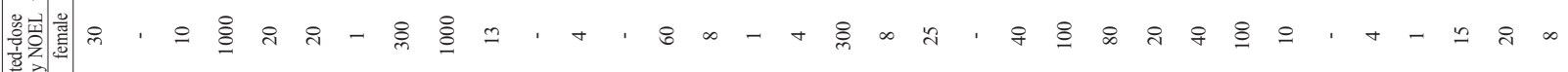

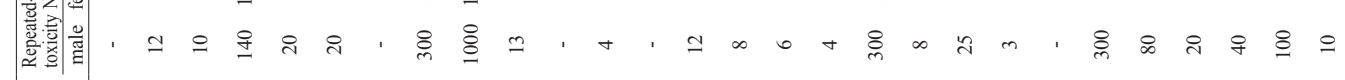

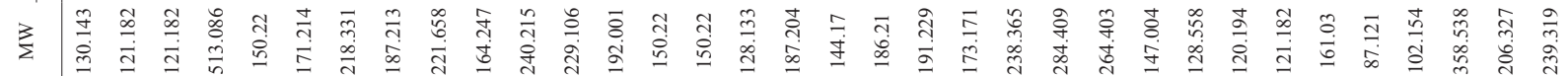

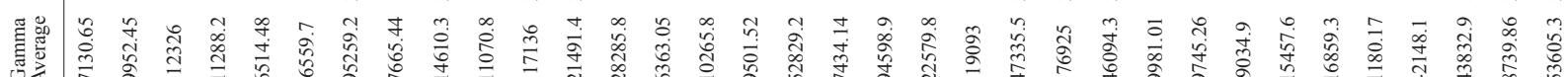

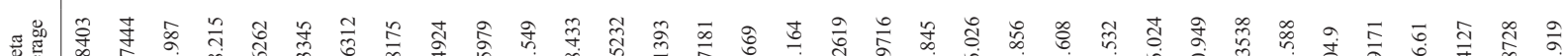

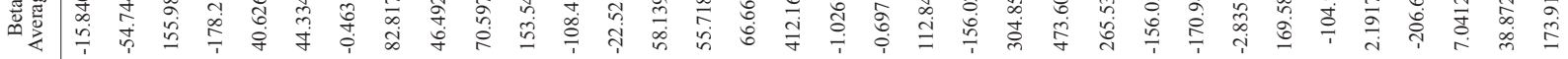

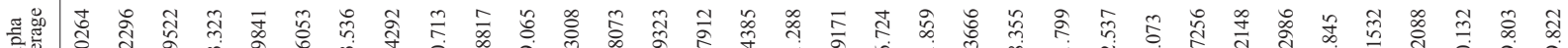

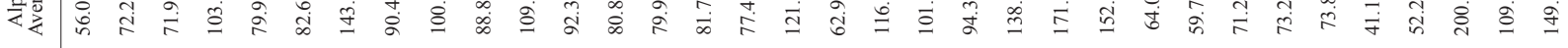

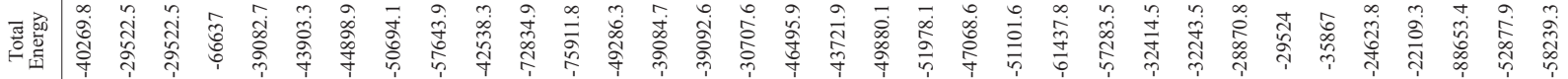

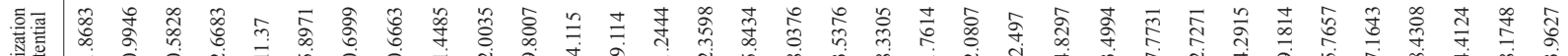

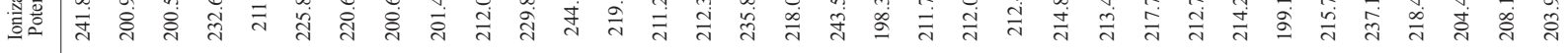

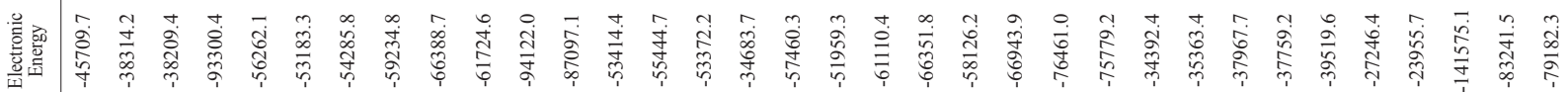

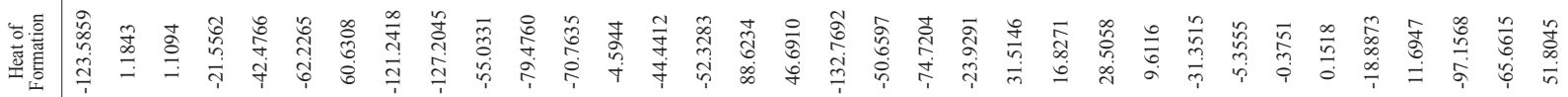

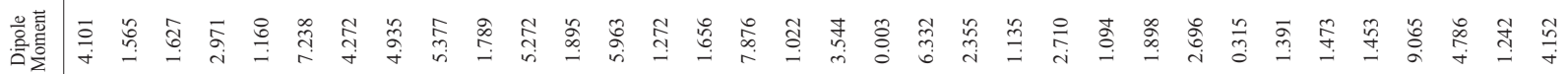

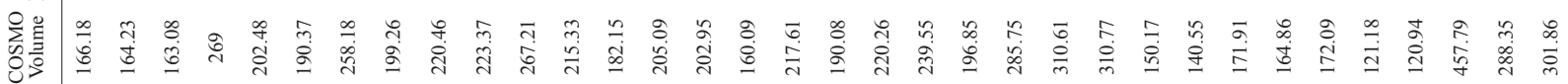

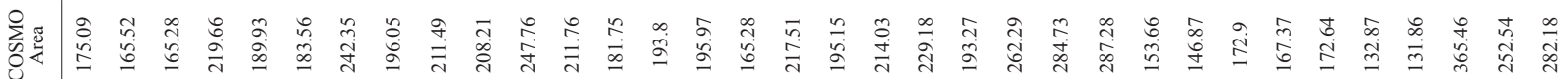

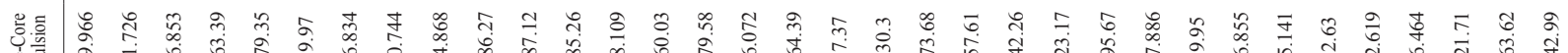

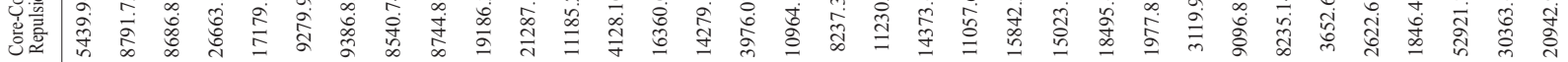
諆 Z 点

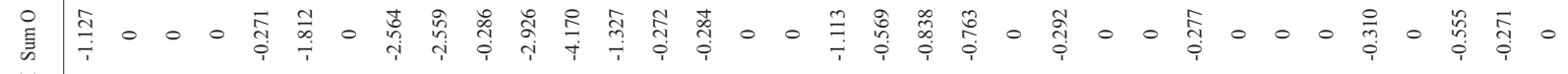
王

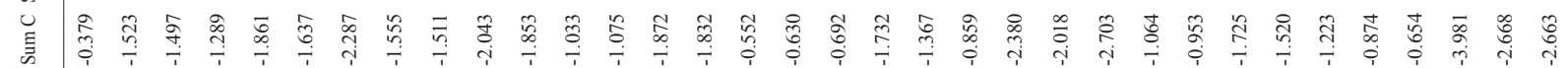

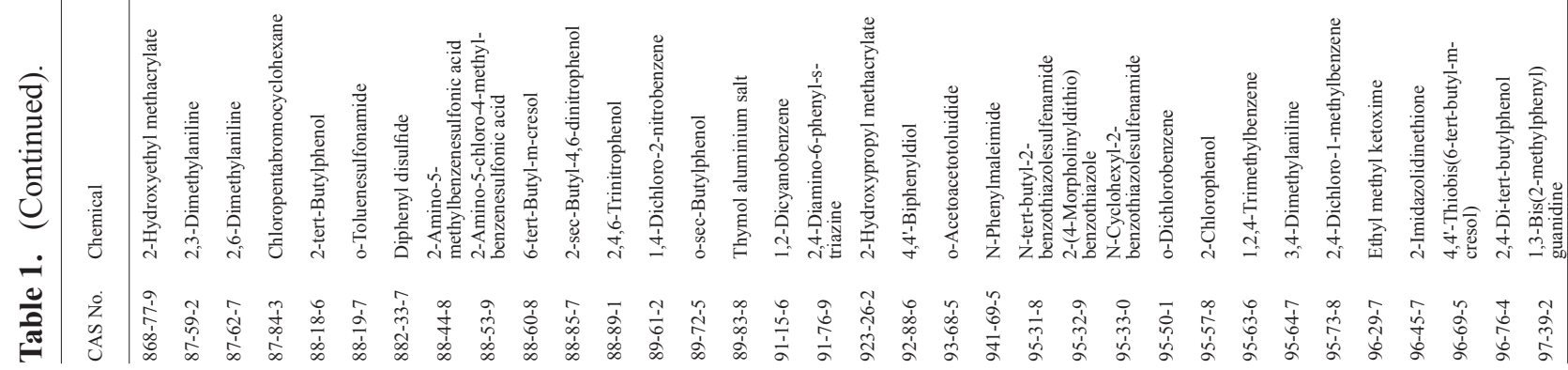


T. Hisaki et al.

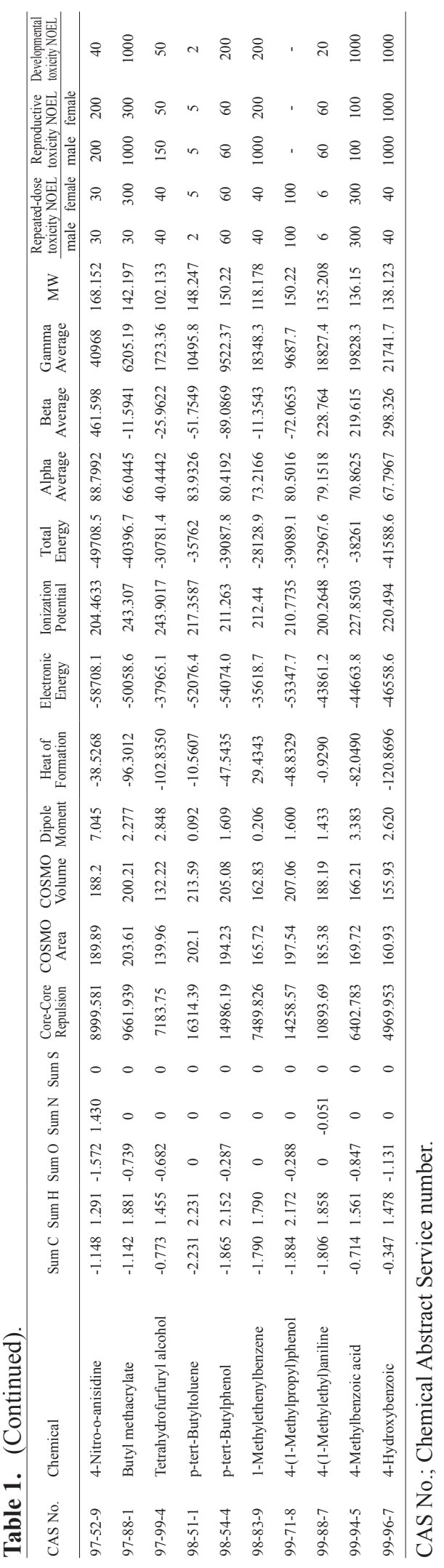

Vol. 40 No. 2 
QSAR prediction of systemic toxicity of cosmetic ingredients

tion was performed and then molecular-orbital descriptors were calculated using the PM3 Hamiltonian of a semi-empirical MO package (MOPAC2002). The calculated descriptors (listed in Table 1) are: Sum of charges of carbon, hydrogen, oxygen, nitrogen, sulphur (Sum C, Sum H, Sum O, Sum N, Sum S), core-core repulsion, COSMO area, COSMO volume, dipole moment, heat of formation, electronic energy, ionization potential, total energy, average of polarizability $\alpha, \beta, \gamma$ (alpha, beta, gamma average), and molecular weight (MW).

These descriptors were grouped by correlation coefficient ( $r$ value; results for the repeated-dose toxicity model are shown in Table 2 as an example), and independent descriptors showing a statistically significant correlation with NOEL (Tables 3-5) were chosen to be used as the input layer for further analysis.

\section{Artificial neural network (ANN) analysis}

Calculated molecular descriptors and in-vivo-determined NOELs obtained from the database were correlated using an artificial neural network. A typical ANN consists of an input layer, a hidden layer, and an output layer, as previously reported (Hirota et al., 2013; Fig. 1). In this study, two hidden layers were used for repeated-dose toxicity and reproductive toxicity, while a single hidden layer was used for developmental toxicity. In-vivo-determined NOEL was the output layer, and the descriptors used for the input layer were correlated with it using the neural network. All calculations were performed using QwikNet Ver.2.23. In ANN learning, an online back-propagation algorithm was used for network training, with values of the learning rate and momentum parameter of 0.02 and 0.7 , respectively. The range of weights was set to be -6 to 6 (weight perturbation; 50\%).

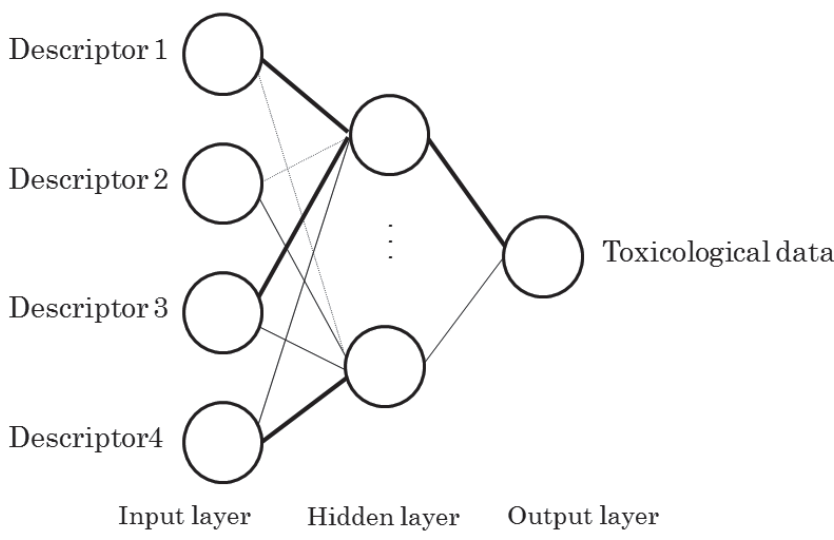

Fig. 1. Schematic representation of a 3-layered neural network.

\section{Ten-fold cross-validation}

The predictive ability of the neural network model was evaluated by means of the 10 -fold cross-validation method (Pasomsub et al., 2010; Hirota et al., 2013). This method systematically removed data successively from the training set. A network model was then constructed on the basis of this reduced data set and used to predict the removed data. This procedure was repeated for all data so that a complete set of predicted values was obtained. The goodness-of-fit was evaluated in terms of root-meansquare (RMS) error, defined as follows:

RMS error $=\sqrt{\sum\left((\text { measuredvalue }- \text { predicted value })^{2}\right) / \text { number of data }}$

\section{Statistical Analysis}

The correlation coefficient ( $r$ value) was determined by the use of Pearson's correlation statistics. Multiple linear regression analysis using the forward selection method was performed by Excel Statistics 2004 software (Social Survey Research Information, Tokyo, Japan).

\section{RESULTS}

\section{Construction and evaluation of repeated-dose toxicity model}

In ANN analysis, the choice of descriptors is very important for acquiring a good-quality model. The use of a large number of descriptors to construct the model improves the predictive performance for the training data, but may impair the predictive performance for test compounds because of the increasing vulnerability of the model. Therefore, descriptors having high similarity were classified into groups, and descriptors that showed statistically significant correlations with NOEL in independent groups were chosen for the input layer of ANN analysis. Table 2 shows the simple correlation coefficients between molecular orbital (MO)-calculated descriptors. Correlation coefficients of less than -0.7 or more than 0.7 are highlighted; these are considered to be high correlations. Then, the correlation between NOEL and the selected MO-calculated descriptors (Table $3 \mathrm{~A}$ ) or the square of the descriptors (Table 3B) was confirmed. Finally, six descriptors (Sum H, Sum N, total energy, heat of formation, gamma average, and ionization potential) belonging to different groups were found to be significant correlated with NOEL, and were selected for use as the input layer of the model.

Because some chemicals have different NOEL for males and females, we used an additional descriptor with values of 0 for males and 1 for females. A preliminary 
T. Hisaki et al.

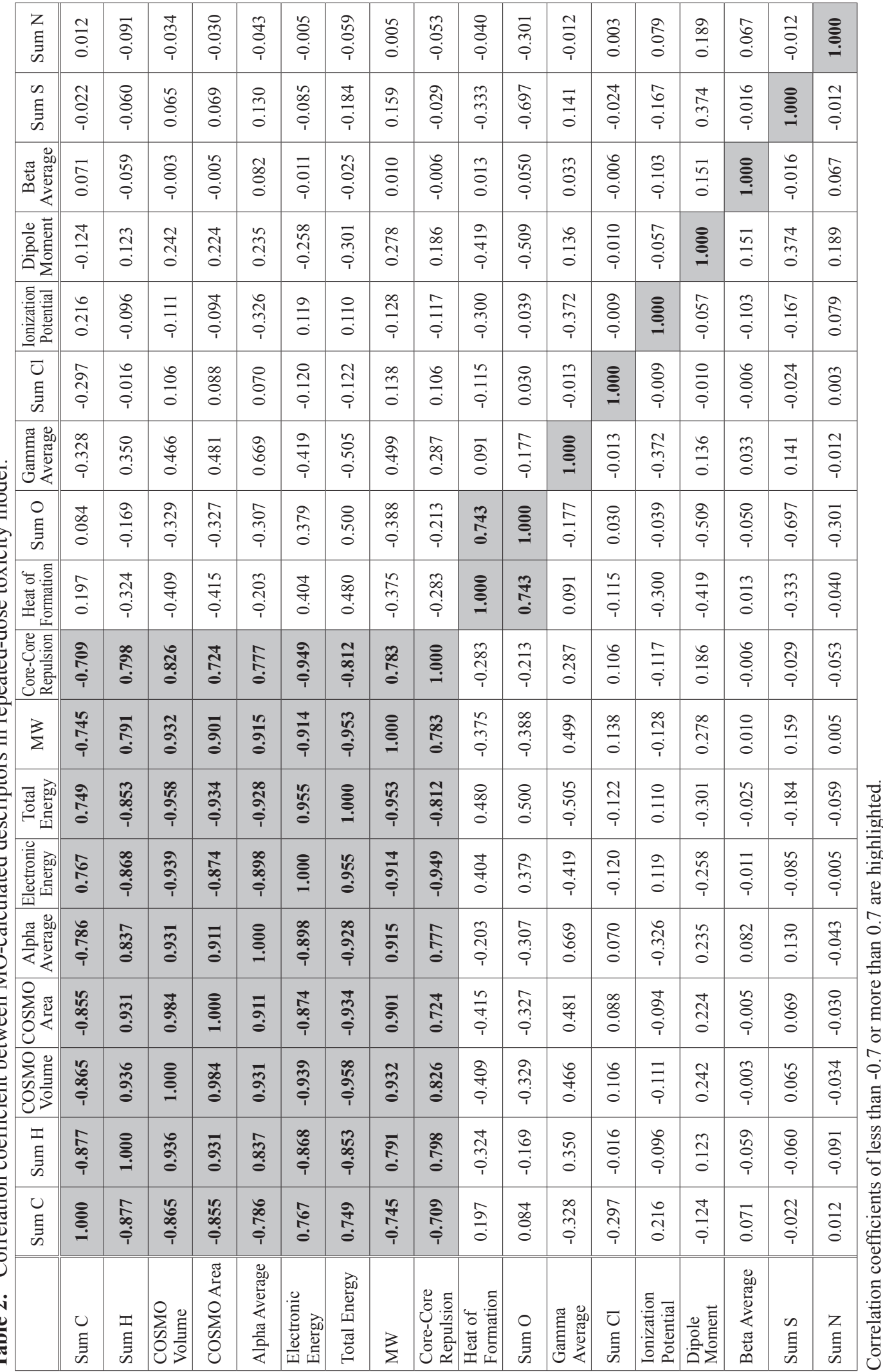


QSAR prediction of systemic toxicity of cosmetic ingredients

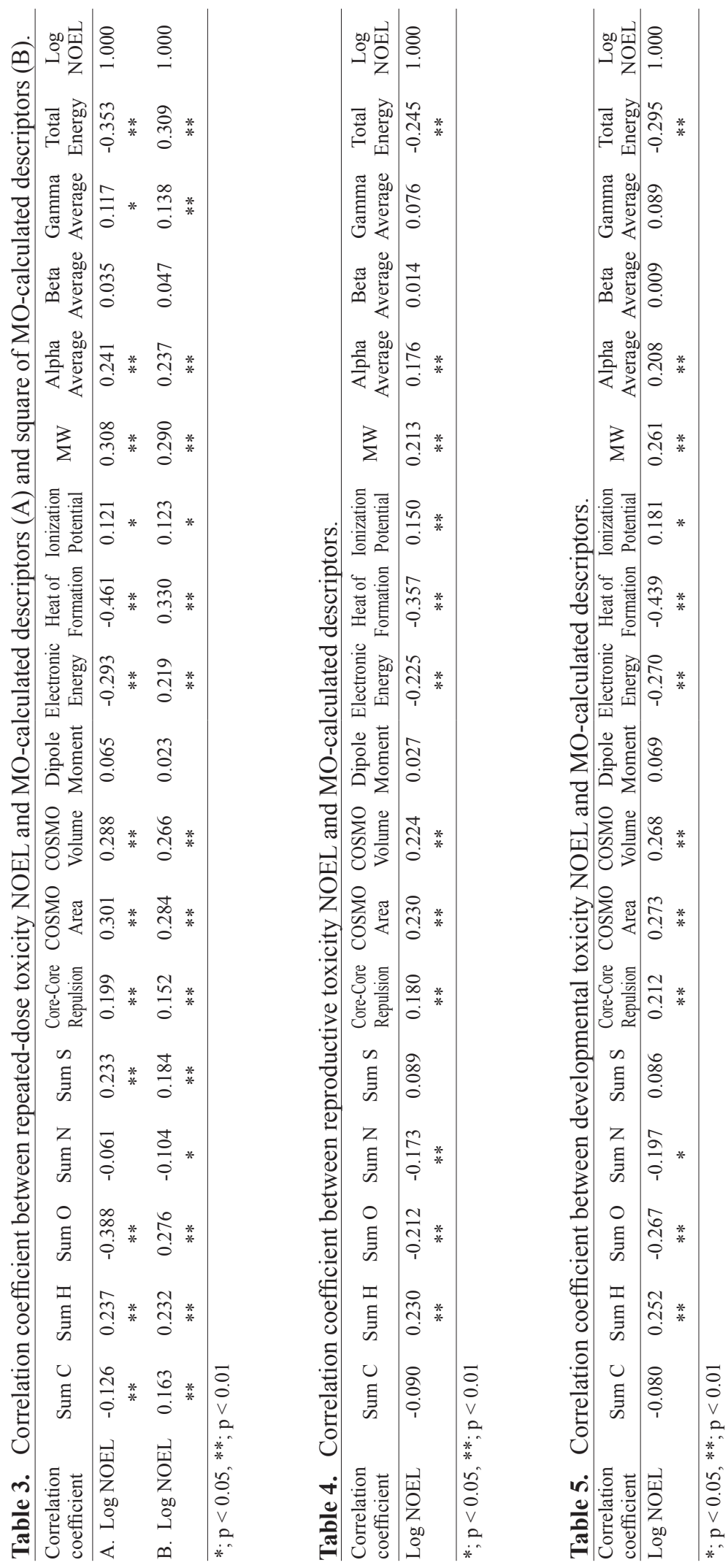




\section{T. Hisaki et al.}

study indicated that the ANN model with a configuration of 7-5-2-1 was optimal in terms of RMS error.

Fig. 2A shows the relationship between observed and calculated NOEL obtained by ANN analysis. The RMS error obtained for the model was 0.507 . The model was then used to predict NOEL for several unknown compounds using a 10 -fold cross validation procedure (Fig. 2B). The predictive RMS error for the 10-fold cross validation analysis was 0.529 .

The accuracy of the predictions is shown in Table 6 . $186(44.2 \%)$ of the predicted NOELs, obtained in 10-fold cross validation, were within a factor of 2 of the in-vivo-determined NOELs, while over $94 \%$ of the chemicals were within a factor of 10 . All chemicals were within a factor of 50 of the in-vivo-determined NOELs.

\section{Construction and evaluation of reproductive toxicity model}

For the reproductive toxicity model, four descriptors (Sum N, total energy, heat of formation, and ionization potential) were selected for the input layer in the same manner as described for the previous model. The correlation between NOEL and MO-calculated descriptors is shown in Table 4. Again, an additional descriptor was employed to distinguish NOELs for males and females. Here, the configuration of the ANN model was 5-5-2-1.

The RMS error obtained for the model was 0.474 (Fig. 3A) and that for the 10-fold cross-validation anal-
(A)

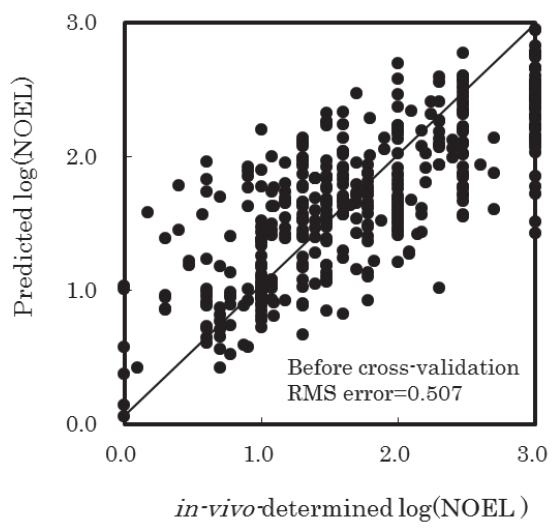

(B)

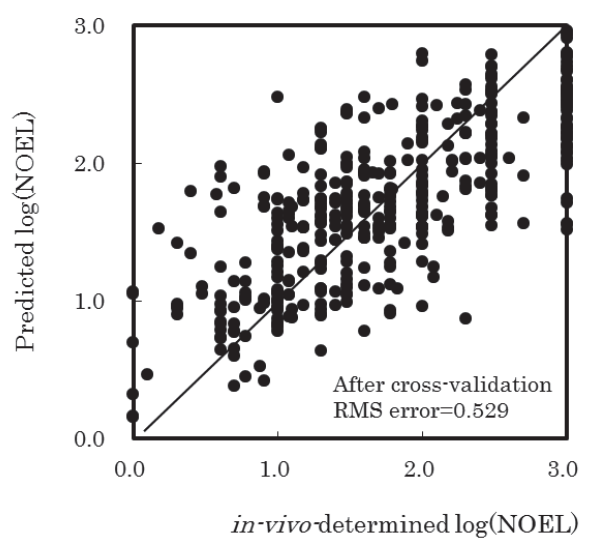

Fig. 2. Correlation between predicted and in-vivo-determined NOEL of repeated-dose toxicity based on MO-descriptors of the dataset of 421 chemicals. (A) Correlation before 10-fold cross validation; (B) After 10-fold cross validation. 6 descriptors selected were used as the input layer and in-vivo-determined NOEL were used as the output layer. The RMS errors of the ANN prediction model were 0.507 (before 10-fold cross validation; (A)) and 0.529 (after 10-fold cross validation; (B)).

(A)

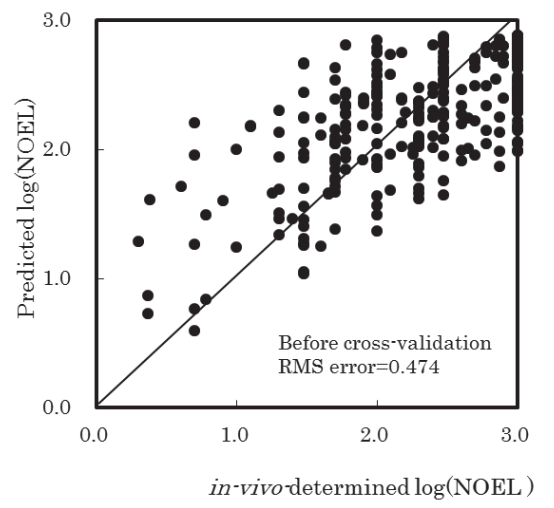

(B)

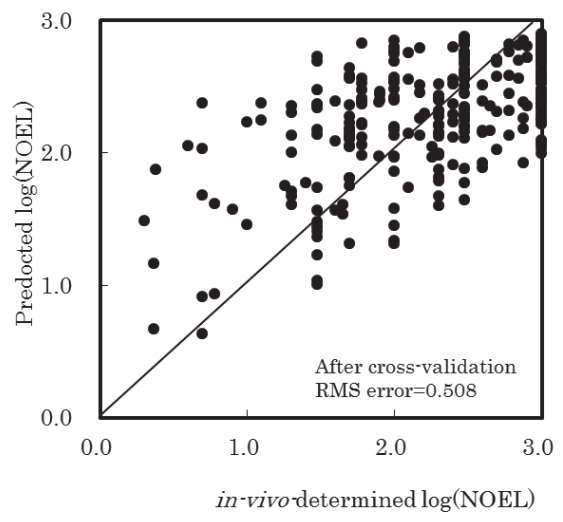

Fig. 3. Correlation between predicted and in-vivo-determined NOEL of reproductive toxicity based on MO-descriptors of the dataset of 315 chemicals. (A) Correlation before 10-fold cross validation; (B) After 10-fold cross validation. 4 descriptors selected were used as the input layer and in-vivo-determined NOEL were used as the output layer. The RMS errors of the ANN prediction model were 0.474 (before 10 -fold cross validation; (A)) and 0.508 (afte r10-fold cross validation; (B)). 
QSAR prediction of systemic toxicity of cosmetic ingredients

ysis was 0.508 (Fig. 3B). As regards accuracy, 144 $(45.7 \%)$ of the predicted NOELs were within a factor of 2 of the in-vivo-determined NOELs, while over $95 \%$ of the chemicals were within a factor of 10 . All chemicals were within a factor of 50 of the in-vivo-determined NOELs (Table 6).

\section{Construction and evaluation of developmental toxicity model}

For the developmental toxicity model, we selected the same four molecular descriptors (Sum N, total energy, heat formation, and ionization potential) as used in the reproductive toxicity model. The correlation between NOEL and the MO-calculated descriptors is shown in Table 5. The configuration of the ANN model was set as 4-4-1.

The RMS error obtained for the model was 0.473 (Fig. 4A) and that for the 10-fold cross-validation analysis was 0.558 (Fig. 4B). As regards accuracy, 58 (37.7\%) of the predicted NOELs were within a factor of 2 of the in-vivo-determined NOELs, while over $94 \%$ of the chemicals were within a factor of 10 (Table 6).

\section{DISCUSSION}

The aim of the present study was to develop an in silico system to predict NOEL values for repeated-dose, developmental, and reproductive toxicities by using the combination of MO calculation and an ANN system. Two commercial packages, TOPKAT (Accelrys) and MolCode Toolboxes (MolCode, Ltd.), are available for predicting LOAELs, but they cannot predict NOELs, which are required for calculation of margin of safety values according to the recommendation of the Scientific Committee on Consumer Safety. Our models are the only ones so far reported that are able to predict NOEL values.

TOPKAT is the most widely used commercial QSAR software package for making quantitative toxicity predictions, and its usefulness has been examined in several studies (Lapenna et al., 2010; Worth et al., 2014).

Table 6. Accuracy of the three models for the prediction of NOEL.

\begin{tabular}{lllllll} 
& \multicolumn{5}{l}{\begin{tabular}{l}
\multicolumn{5}{l}{ Number $(\%)$ of chemicals for which predicted NOELs were compared to in-vivo-determined NOELs } \\
\cline { 2 - 7 }
\end{tabular}} & \multicolumn{1}{l}{ Factor ${ }^{\text {a }}$} \\
\cline { 2 - 7 } & 2 & 5 & 10 & 50 & 100 & Total \\
\hline Model & $186(44.2)$ & $157(37.3)$ & $54(12.8)$ & $24(5.7)$ & $0(0.0)$ & 421 \\
Repeated-dose toxicity & $144(45.7)$ & $123(39.0)$ & $34(10.8)$ & $14(4.4)$ & $0(0.0)$ & 315 \\
Reproductive toxicity & $58(37.2)$ & $69(44.2)$ & $21(13.5)$ & $6(3.8)$ & $2(1.3)$ & 156 \\
Developmental toxicity &
\end{tabular}

a) Factor means a range of $1 / 2$ to 2 times, $1 / 5$ to 5 times, $1 / 10$ to 10 times, $1 / 50$ to 50 times and $1 / 100$ to 100 times.

(A)

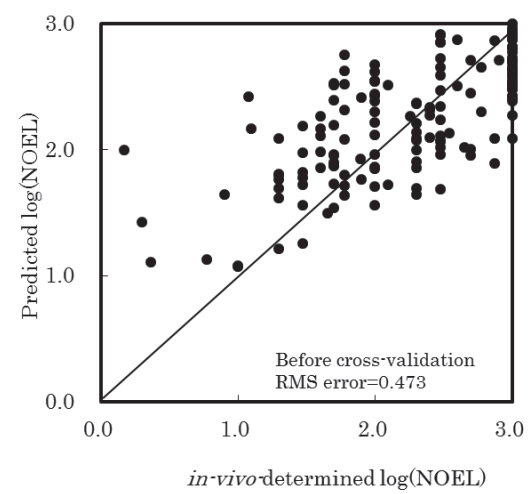

(B)

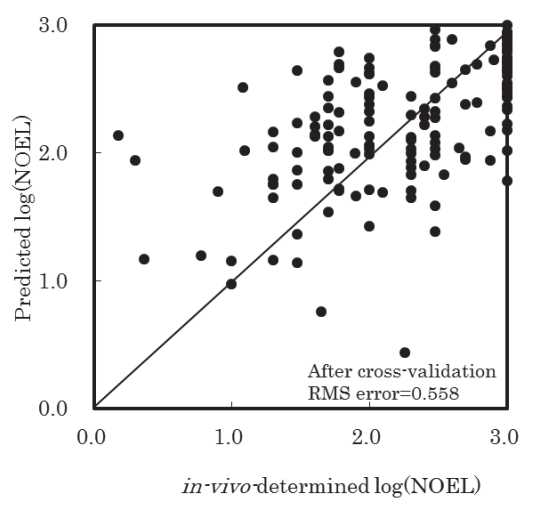

Fig. 4. Correlation between predicted and in-vivo-determined NOEL of developmental toxicity based on MO-descriptors of the dataset of 156 chemicals. (A) Correlation before 10-fold cross validation; (B) After 10-fold cross validation. 4 descriptors selected were used as the input layer and in-vivo-determined NOEL were used as the output layer. The RMS errors of the ANN prediction model were 0.473 (before 10-fold cross validation; (A)) and 0.558 (after 10-fold cross validation; (B)). 


\section{T. Hisaki et al.}

External validation is desirable to estimate the true predictive power of a QSAR model (Kubinyi et al., 1998; Veerasamy et al., 2011), and this was done by Venkatapathy et al. (2004) using a set of 160 chemicals that were not present in TOPKAT's database, collected from the U.S. EPA's Office of Pesticide Programs (OPP). The percentage of LOAELs predicted accurately to within factors of 2, 5, and 10 of the experimental values were 34,57 , and $72 \%$, respectively and similar statistics were obtained for another set of chemicals from a different database. In the present study, we used 10-fold cross-validations to test the model, and the percentages of repeated-dose toxicity NOELs predicted accurately to within factors of 2, 5, and 10 of the experimental values were $44.2,81.5$, and $94.2 \%$, respectively. Similar results were obtained with our reproductive toxicity and developmental toxicity models (Table 6). Since our validation method was different from that used the study in Venkatapathy et al. (2004) direct comparison is not appropriate, but our models appear to offer superior predictive accuracy.

It is important to note that if certain one- or two-atom fragments are missing in TOPKAT's LOAEL model substructural library for any given chemical, or if the fragments are not adequately covered by the chemicals in the model database, TOPKAT issues a warning stating that a prediction cannot be made or may be unreliable. In one of the studies by Venkatapathy et al. (2004), TOPKAT was unable to predict LOAEL of 134 (39\%) out of 343 tested chemicals, while $53(18 \%)$ out of 287 tested chemicals were unpredictable in the study by Tilaoui et al. (2007) On the other hand, our models were able to achieve similar or superior accuracy without "removing" any test chemical. Overall, our present results indicate that our models can predict NOELs of a wide range of chemicals with reasonable accuracy and high robustness.

We extracted six molecular descriptors (Sum H, Sum N, total energy, heat of formation, gamma average, and ionization potential) for the repeated-dose toxicity model and four (Sum N, total energy, heat of formation, and ionization potential) for the developmental and reproductive toxicity models. Although pathways of toxicity are complex, and the contribution of each descriptor is unclear, at least a qualitative discussion is possible. For example, liposolubility and stability are important physicochemical properties of chemicals. Liposolubility is influenced by polarizability, molecular volume and hydrogen-bonding property. Therefore, sum H, sum N, and ionization potential, which are relevant to hydrogen-bonding property, are expected to be available as descriptors of liposolubility. In turn, liposolubility influences intestinal absorption, binding affinity to proteins and receptors, and histological dis- tribution and accumulation in general. Therefore, descriptors of liposolubility should provide information relevant to these biological processes. Further, ionization potential, heat of formation, and total energy are indicators of molecular energy, which would determine molecular stability, and this in turn would be related to metabolic degradation. Sum $\mathrm{H}$, sum $\mathrm{N}$ and ionization potential are also related to electronegativity, and therefore to reactivity in nucleophilic and electrophilic reactions. In other words, the descriptors extracted in this study seem relevant to biological reactions that are expected to have central roles in the mechanisms and pathways of toxicity (Fig. 5).

One of the reasons for the limited success so far in developing QSAR for systemic toxicity is the lack of promising datasets that can be used to train QSAR models, as reviewed elsewhere (Piparo and Worth, 2010; Lapenna et al., 2010). Since the performance of a QSAR model depends on the numerical values of the NOELs used in training the model (Leonard and Roy, 2006), training of a QSAR model for assessing the toxicity of cosmetic ingredients should ideally be done with the aid of datasets of potential or actual cosmetic ingredients (e.g. chemicals from the International Nomenclature of Cosmetic Ingredients (INCI) list). Since toxicological data for such chemicals are quite limited, all the datasets available in the JECDB were included in this study. However, among the 245 chemicals included in this study, 50 chemicals were listed in INCI. When comparison analysis was conducted with only these 50 chemicals, the percentage of repeated-dose toxicity NOELs predicted to within factors of 2, 5, and 10 of the experimental values were 33.3, 77.0, and 96.5\%, respectively (Table 7). The predictions for chemicals listed in INCI compared well with those for all chemicals from JECDB (Table 6), and thus we consider that the present models are applicable to a broad range of chemicals, including cosmetic ingredients.

Another important issue is that NOELs for developmental toxicity collected in this study were obtained from toxicity tests in which observation was conducted only from the day of birth up to the 4th day. Therefore, toxicities that may appear before or after this period, which includes teratogenicity, cannot be assessed in our model. Needless to say, improvements are needed in the systemic toxicity database.

We consider that our present findings represent a proofof-concept for prediction of NOEL of chemicals using QSAR models. This is a major step towards calculation of margin of safety values. Further, when limited data are available to estimate the toxicity of a chemical of interest, it would enable us to read across from corresponding data for suitable analogues. However, it is important 
QSAR prediction of systemic toxicity of cosmetic ingredients

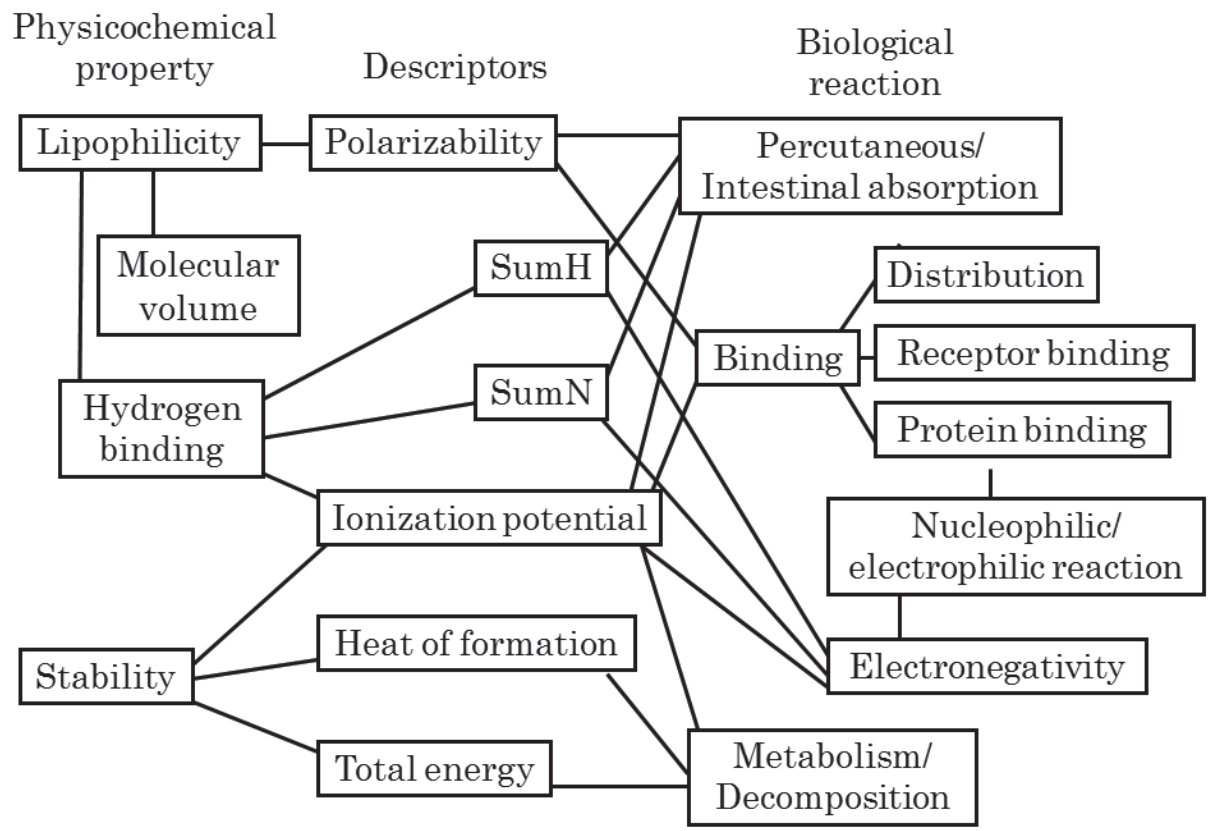

Fig. 5. Relationships between selected descriptors and toxicity-related processes.

Table 7. Accuracy of the three models for the prediction of NOEL for the subset of chemicals included in the INCI list. Number (\%) of INCI-listed chemicals for which predicted NOELs were compared to the invivo-determined NOELs

\begin{tabular}{lllllll} 
& \multicolumn{1}{l}{ Factor a) } & & & & \\
\cline { 2 - 7 } & 2 & 5 & 10 & 50 & 100 & Total \\
\hline Model & $29(33.3)$ & $38(43.7)$ & $17(19.5)$ & $3(3.4)$ & $0(0.0)$ & 87 \\
Repeated-dose toxicity & $44(53.7)$ & $31(37.8)$ & $5(6.1)$ & $2(2.4)$ & $0(0.0)$ & 82 \\
Reproductive toxicity & $19(46.3)$ & $17(41.5)$ & $4(9.8)$ & $1(2.4)$ & $0(0.0)$ & 41 \\
Developmental toxicity &
\end{tabular}

a) Factor means a range of $1 / 2$ to 2 times, $1 / 5$ to 5 times, $1 / 10$ to 10 times, $1 / 50$ to 50 times and $1 / 100$ to 100 times.

to realize that toxicity occurs through multiple complex mechanisms, so QSAR predictions cannot entirely replace experiments. The most promising predictive methodology may be a weight of evidence approach, combining in silico analysis with in vitro methods.

Conflict of interest---- The authors declare that there is no conflict of interest.

\section{REFERENCES}

Brown, N.A., Spielmann, H., Bechter, R., Flint, O.P., Freeman, S.J. and Jelinek, R.J. (1995): Screening chemicals for reproductive toxicity: The current alternatives; the report and recommendations of an ECVAM. Altern. Lab. Anim., 23, 868-882.
Cronin, M.T.D. and Worth, A.P. (2008): (Q)SARs for predicting effects relating to reproductive toxicity. QSAR Comb. Sci., 27, 91-100.

Hartung, T. and Rovida, C. (2009): Chemical regulators have overreached. Nature, 460, 1080-1081.

Hirota, M., Kouzuki, H., Ashikaga, T., Sono, S., Tsujita, K., Sasa, H. and Aiba, S. (2013): Artificial neural network analysis of data from multiple in vitro assays for prediction of skin sensitization potency of chemicals. Toxicol. In Vitro, 27, 1233-1246.

Jagt, K., Munn, S., Tørsløv, J. and Bruijn, J. (2004): Alternative approaches can reduce the use of test animals under REACH. Report EUR 21405.

Kubinyi, H., Hamprecht, F.A. and Mietzner, T. (1998): Three-dimensional quantitative similarity-activity relationships $(3 \mathrm{D}$ QSiAR) from SEAL similarity matrices. J. Med. Chem., 41, 2553-2564.

Lapenna, S., Fuart-Gatnik, M. and Worth, A. (2010): Review of 


\section{T. Hisaki et al.}

QSAR models and software tools for predicting acute and chronic systemic toxicity. JRC Scientific and Technical report., EUR $24639 \mathrm{EN}$.

Leonard, J.T. and Roy, K. (2006): On selection of training and test sets for the development of predictive QSAR models. QSAR Comb. Sci., 25, 235-251.

Mumtaz, M.M., Knauf, L.A., Reisman, D.J., Peirano, W.B., DeRosa, C.T., Gombar, V.K., Enslein, K., Carter, J.R., Blake, B.W., Huque, K.I. and Ramanujam, V.M.S. (1995): Assessment of effect levels of chemicals from quantitative structure-activity relationship (QSAR) models. I. Chronic lowest-observed-adverse-effect level (LOAEL). Toxicol. Lett., 79, 131-143.

Pasomsub, E., Sukasem, C., Sungkanuparph, S., Kijsirikul, B. and Chantratita, W. (2010): The application of artificial neural networks for phenotypic drug resistance prediction: evaluation and comparison with other interpretation systems. Jpn. J. Infect. Dis., 63, 87-94.

Piparo, E.L. and Worth, A. (2010): Review of QSAR models and software tools for predicting developmental and reproductive toxicity. JRC Scientific and Technical Report, EUR 24522 EN.

Rupp, B., Appel, K.E. and Gundert-Remy, U. (2010): Chronic oral LOAEL prediction by using a commercially available computational QSAR tool. Arch. Toxicol., 84, 681-688.

Seiler, A.E. and Spielmann, H. (2011): The validated embryonic stem cell test to predict embryotoxicity in vitro. Nat. Protoc., 6, 961-978.

Spielmann, H., Pohl, I., Döring, B., Liebsch, M. and Moldenhauer,
F. (1997): The embryonic stem cell test (EST), an in vitro embryotoxicity test using two permanent mouse cell lines: 3T3 fibroblasts and embryonic stem cells. In Vitro Toxicol., 10, 119-127.

Spielmann, H. and Vogel, R. (2006): REACH testing requirements must not be driven by reproductive toxicity testing in animals. Altern. Lab. Anim., 34, 365-366.

Tilaoui, L., Schilter, B., Tran, L.A., Mazzatorta, P. and Grigorov, M. (2007): Integrated computational methods for prediction of the lowest observable adverse effect level of food-borne molecules. QSAR Comb. Sci., 26, 102-108.

Veerasamy, R., Rajak, H., Jain, A., Sivadasan, S., Varghese, C.P. and Agrawal, R.K. (2011): Validation of QSAR models - Strategies and importance. International Journal of Drug Design \& Discovery, 3, 511-519.

Venkatapathy, R., Moudgal, C.J. and Bruce, R.M. (2004): Assessment of the oral rat chronic lowest observed adverse effect level model in TOPKAT, a QSAR software package for toxicity prediction. J. Chem. Inf. Comput. Sci., 44, 1623-1629.

Worth, A., Barroso, J., Bremer, S., Burton, J., Casati, S., Coecke, S., Corvi, R., Desprez, B., Dumont, C., Gouliarmou, V., Goumenou, M., Gräpel, R., Griesinger, C., Halder, M., Janusch, A., Kienzler, A., Madia, F., Munn, S., Nepelska, M., Paini, A., Price, A., Prieto, P., Rolaki, A., Schäffer, M., Triebe, J., Whelan, M., Wittwehr, C. and Zuang, V. (2014): Alternative methods for regulatory toxicology - a state-of-the-art review. JRC Scientific and Technical Report, EUR 26797 EN. 